\title{
The normal Type la SN 2003hv out to very late phases ${ }^{\star \star \star \star}$
}

\author{
G. Leloudas ${ }^{1}$, M. D. Stritzinger ${ }^{2,1}$, J. Sollerman ${ }^{1,3}$, C. R. Burns ${ }^{4}$, C. Kozma ${ }^{3}$, K. Krisciunas ${ }^{5}$, J. R. Maund ${ }^{1}$, P. Milne ${ }^{6}$,
} A. V. Filippenko ${ }^{7}$, C. Fransson 3 , M. Ganeshalingam ${ }^{7}$, M. Hamuy ${ }^{8}$, W. Li ${ }^{7}$, M. M. Phillips ${ }^{2}$, B. P. Schmidt ${ }^{9}$, J. Skottfelt ${ }^{10}$, S. Taubenberger ${ }^{11}$, L. Boldt ${ }^{2}$, J. P. U. Fynbo ${ }^{1}$, L. Gonzalez ${ }^{8}$, M. Salvo ${ }^{9}$, and J. Thomas-Osip ${ }^{2}$

1 Dark Cosmology Centre, Niels Bohr Institute, University of Copenhagen, Juliane Maries Vej 30, 2100 Copenhagen $\emptyset$, Denmark e-mail: giorgos@dark-cosmology.dk

2 Las Campanas Observatory, Carnegie Observatories, Casilla 601, La Serena, Chile

3 The Oskar Klein Centre, Department of Astronomy, Stockholm University, AlbaNova, 10691 Stockholm, Sweden

4 Observatories of the Carnegie Institution of Washington, 813 Santa Barbara St., Pasadena, CA 91101, USA

5 Department of Physics, Texas A\&M University, College Station, TX 77843, USA

${ }^{6}$ Department of Astronomy and Steward Observatory, University of Arizona, Tucson, AZ 85721, USA

7 Department of Astronomy, University of California, Berkeley, CA 94720-3411, USA

8 Departamento de Astronomía, Universidad de Chile, Casilla 36-D, Santiago, Chile

9 Research School of Astronomy and Astrophysics, Australian National University, Cotter Road, Weston Creek, PO 2611, Australia

10 Niels Bohr Institute, University of Copenhagen, Blegdamsvej 17, 2100 Copenhagen Ø, Denmark

11 Max-Planck-Institut für Astrophysik, Karl-Schwarzschild-Str. 1, 85741 Garching bei München, Germany

Received 21 April 2009 / Accepted 7 July 2009

\section{ABSTRACT}

Aims. We study a thermonuclear supernova ( $\mathrm{SN})$, emphasizing very late phases.

Methods. An extensive dataset for SN 2003hv that covers the flux evolution from maximum light to day +786 is presented. This includes 82 epochs of optical imaging, 24 epochs of near-infrared (NIR) imaging, and 10 epochs of optical spectroscopy. These data are combined with published nebular-phase IR spectra, and the observations are compared to model light curves and synthetic nebular spectra.

Results. SN 2003hv is a normal Type Ia supernova (SN Ia) with photometric and spectroscopic properties consistent with its rarely observed $B$-band decline-rate parameter, $\Delta m_{15}(B)=1.61 \pm 0.02$. The blueshift of the most isolated [Fe II] lines in the nebular-phase optical spectrum appears consistent with those observed in the IR at similar epochs. At late times there is a prevalent color evolution from the optical toward the NIR bands. We present the latest-ever detection of a SN Ia in the NIR in Hubble Space Telescope images. The study of the ultraviolet/optical/infrared (UVOIR) light curve reveals that a substantial fraction of the flux is "missing" at late times. Between 300 and 700 days past maximum brightness, the UVOIR light curve declines linearly following the decay of radioactive ${ }^{56} \mathrm{Co}$, assuming full and instantaneous positron trapping. At 700 days we detect a possible slowdown of the decline in optical-bands, mainly in the $V$-band.

Conclusions. The data are incompatible with a dramatic infrared catastrophe (IRC). However, the idea that an IRC occurred in the densest regions before 350 days can explain the missing flux from the UVOIR wavelengths and the flat-topped profiles in the NIR. We argue that such a scenario is possible if the ejecta are clumpy. The observations suggest that positrons are most likely trapped in the ejecta.

Key words. supernovae: general - supernovae: individual: SN 2003hv

\section{Introduction}

Studying the late-phase emission of Type Ia supernovae (hereafter SNe Ia) provides an excellent opportunity to elucidate the physical nature of these thermonuclear explosions. With late-time observations it is in principle possible to constrain the nucleosynthetic yields and the distribution of elements (Kozma et al. 2005; Motohara et al. 2006; Stritzinger et al. 2006b; Mazzali et al. 2007), the magnetic field configuration of the ejecta and hence of the progenitor (Colgate et al. 1980;

\footnotetext{
* Based on observations collected at the European Southern Observatory, Paranal, Chile (ESO Programmes 073.D-0294(AB), 074.D-0259(BC) and 075.D-0116(AB)), Cerro Tololo Inter-American Observatory, Lick Observatory, Las Campanas Observatory, Siding Spring Observatory, and the Hubble Space Telescope.

$\star \star$ Tables 3, 4, and Appendix A are only available in electronic form at http://www . aanda.org
}

Ruiz-Lapuente \& Spruit 1998; Milne et al. 1999), and potentially their contribution to the diffuse Galactic $511 \mathrm{keV}$ line (Milne et al. 1999; Prantzos 2008).

Previous studies of the late-time emission of SNe Ia have demonstrated the increased importance of the near-infrared (NIR) emission. This investigation builds upon earlier studies of SN 2000cx (Sollerman et al. 2004, hereafter S04) and SN 2001el (Stritzinger \& Sollerman 2007, hereafter SS07) but extends to even later phases. We present observations of SN 2003hv that cover 786 days past $B$-band maximum $\left(B_{\max }\right)$. One of the main motivations of this study was to investigate whether an infrared catastrophe (IRC) occurs in the ejecta at these very late phases. The IRC is a thermal instability, predicted by Axelrod (1980), which shifts the bulk of the emission from the optical and NIR regime to the mid- and far-IR fine-structure lines once the temperature in the ejecta falls below a critical limit. While the IRC has been proposed to explain the line evolution of SN 1987A 
(Spyromilio \& Graham 1992; Kozma \& Fransson 1998a), it has never been observed for a thermonuclear SN (see, however, Maeda et al. 2009a, on the peculiar SN 2006gz). Models suggest that the IRC should commence, depending on the structure and composition of the ejecta, sometime between 500 and 700 days past maximum brightness and become apparent with a dramatic drop in the optical and NIR luminosity. This corresponds to shortly after the end of our previous multi-band observations of other SNe Ia (S04; SS07).

SN 2003hv was discovered by LOTOSS (Beutler \& Li 2003) on 9.5 September 2003 (UT dates are used throughout this paper). It was located $17^{\prime \prime}$ east and $57^{\prime \prime}$ south of the nucleus of the S0 galaxy NGC 1201. Dressler et al. (2003) classified it as a SN Ia the day after discovery. SN $2003 \mathrm{hv}$ reached an apparent $B$-band magnitude of $12.45 \mathrm{mag}$, thus being the brightest supernova discovered in 2003, and one of the brightest SNe Ia observed over the past decade. NGC 1201 has a direct distance measurement that is based on the surface brightness fluctuations method (SBF; Tonry et al. 2001). By applying a correction of $-0.16 \mathrm{mag}$ (Jensen et al. 2003), the distance modulus of NGC 1201 is $\mu_{\mathrm{SBF}}=31.37 \pm 0.30 \mathrm{mag}$, corresponding to a distance of $18.79 \pm 2.60 \mathrm{Mpc}$. The Galactic extinction in the direction of NGC 1201 is low, $E(B-V)=0.016$ mag (Schlegel et al. 1998), and we show below that there is no evidence of any host-galaxy extinction. As SN 2003hv suffered little extinction, was located in the outskirts of its host, and was bright, it made an excellent target for our late-time photometric ( $U B V R I J H K$ ) and spectroscopic (optical) observational campaign.

SN 2003hv has already attracted some attention in the literature. Motohara et al. (2006) published a NIR spectrum taken 394 days past maximum brightness. They found that the [Fe II] $1.644 \mu \mathrm{m}$ emission line exhibited a flat-topped profile and was blueshifted by $\sim 2600 \mathrm{~km} \mathrm{~s}^{-1}$. Gerardy et al. (2007) showed a mid-infrared (MIR) spectrum $(5.2-15.2 \mu \mathrm{m})$ of SN 2003hv taken on day +358 with the InfraRed Spectrograph onboard the Spitzer Space Telescope. From the observed [Co III] emission they estimated that $\sim 0.5 M_{\odot}$ of ${ }^{56} \mathrm{Ni}$ was synthesized in the explosion. In this paper, we present a comprehensive study of SN 2003hv that covers the flux evolution from early to very late phases. Some preliminary results of our study were presented by Leloudas et al. (2009). Here, we have used more optical and NIR earlytime data, have re-analyzed all data in a consistent manner, and have supplemented our late-time analysis with data from the Hubble Space Telescope (HST) and spectral-synthesis modeling; we have also included the spectra published by Motohara et al. (2006) and Gerardy et al. (2007).

The organization of this paper is as follows. Section 2 presents the observations that were collected using ground-based facilities and HST, and Sect. 3 describes the corresponding data reduction. The results are described in Sect. 4. Section 5 provides a discussion, and the main conclusions are summarized in Sect. 6.

\section{Observations}

The observations presented in this paper were conducted at optical and NIR wavelengths with a variety of facilities. A brief description of the observations is given below. Observing logs for our data are found in Table 1 and in Tables A.1-A.3 of the Appendix.

\subsection{Cerro Tololo observations}

Optical photometry of SN 2003hv was obtained with the Cassegrain Direct Imager attached to the 0.9-m telescope located at the Cerro Tololo Inter-American Observatory (CTIO). Ten epochs of $U B V R I$ imaging were obtained from 1 to 74 days past $B_{\max }$. In addition, the CTIO 1.3-m telescope equipped with ANDICAM was used to collect 15 epochs of early-phase $Y J H K_{s}$ photometry that covers the flux evolution from 1 to 62 days past $B_{\max }$.

\subsection{KAIT observations}

The 0.76-m Katzman Automatic Imaging Telescope (KAIT; Filippenko et al. 2001) observed SN 2003hv in BVRI over the course of 42 epochs. These observations were obtained beginning 1 day after $B_{\max }$ and extend to +135 days.

\subsection{Las Campanas observations}

Thirteen epochs of optical photometry covering 10-109 days past $B$ maximum were obtained with the Swope 1-m telescope at Las Campanas Observatory (LCO) during the course of the Carnegie Type II Supernova (CATS) program (Hamuy et al. 2009). These images were obtained with a set of $U B V R I$ filters and a SITe3 detector. Four optical spectra were obtained at LCO from 1 to 17 days past maximum. A journal of the spectroscopic observations is provided in Table 1 .

\subsection{Siding Spring observations}

The European Supernova Collaboration (ESC; see, e.g., Benetti et al. 2004), as part of a European Research Training Network on the study of SNe Ia ${ }^{1}$, obtained four optical spectra and five epochs of photometry at the Siding Spring Observatory (SSO). These data were collected with the Imager and the Double Beam Spectrograph (DBS) on the 2.3-m telescope. They cover the intermediate phases from 52 to 202 days past maximum.

\subsection{VLT observations}

The Very Large Telescope (VLT) was primarily used to obtain late-epoch observations in the optical and NIR. With the exception of one epoch of optical imaging and spectroscopy obtained on day +73 , the VLT observations were conducted from day +320 to +767 . This portion of the study complements and extends our previous studies of SN 2000cx (S04) and SN 2001el (SS07) by over 200 days. $U$ - and $K_{s}$-band observations, however, were only carried out to day $\sim 540$.

Optical imaging was performed with the FOcal Reducer and low dispersion Spectrographs (FORS1 and FORS2) mounted on the Antu (VLT - UT1) and Kueyen (VLT - UT2) telescopes. These observations were obtained in service mode under favorable conditions. Details can be found in Table A.1. The optical data can be divided into three main epochs: approximately 340 , 520 , and 700 days past maximum light. No $B$-band imaging was conducted during the middle epoch.

NIR imaging $\left(J_{s} H K_{s}\right)$ was performed with ISAAC (Infrared Spectrometer And Array Camera) mounted on the Antu telescope. Imaging was obtained in the short-wavelength mode using a jitter-offset technique. To facilitate a multi-wavelength study, the NIR images were taken at epochs similar to those of the optical images. A log is presented in Table A.2.

On day +320 a nebular spectrum was taken with FORS1. Exposures were obtained with the $300 \mathrm{~V}$ and $300 \mathrm{I}$ grisms, and an

\footnotetext{
1 wwW.mpa-garching.mpg.de/ rtn
} 
Table 1. Log of spectroscopy.

\begin{tabular}{cccllcc}
\hline \hline $\begin{array}{c}\text { Date } \\
(\mathrm{UT})\end{array}$ & $\begin{array}{c}\text { MJD } \\
(\text { days })\end{array}$ & $\begin{array}{c}\text { Phase }^{a} \\
(\text { days })\end{array}$ & Telescope & Instrument & $\begin{array}{c}\text { Resolution } \\
(\AA)\end{array}$ & $\begin{array}{c}\text { Exposure time } \\
(\mathrm{s})\end{array}$ \\
\hline 20030910 & 52892.35 & 1.2 & LCO Baade & IMACS & 23.0 & $2 \times 300$ \\
20030915 & 52897.34 & 6.1 & LCO Clay & LDSS2 & 13.5 & $1 \times 60$ \\
20030918 & 52900.39 & 9.2 & LCO duPont & ModSpec & 6.0 & $2 \times 300$ \\
20030926 & 52908.31 & 17.1 & LCO duPont & WFCCD & 6.0 & $2 \times 300$ \\
20031030 & 52942.6 & 51.4 & SSO 2.3 m & DBS & 4.8 & $2 \times 900^{b}$ \\
20031121 & 52964.25 & 73.1 & VLT Antu & FORS1 & 11.5 & $2 \times 300^{c}$ \\
20031128 & 52971.69 & 80.5 & SSO 2.3 m & DBS & 4.8 & $2 \times 1200^{b}$ \\
20031228 & 53001.53 & 110.3 & SSO 2.3 m & DBS & 4.8 & $2 \times 1200^{b}$ \\
20040130 & 53034.45 & 143.2 & SSO 2.3 m & DBS & 4.8 & $2 \times 1200^{b}$ \\
20040725 & 53211.37 & 320.2 & VLT Kueyen & FORS1 & 11.5 & $4 \times 1200^{c}$ \\
\hline
\end{tabular}

${ }^{a}$ In all tables, "phase" refers to days past $B_{\max }$, which occurred at MJD $=52891.20 \pm 0.30$.

${ }^{b}$ One exposure in the blue arm and one in the red arm.

${ }^{c}$ Exposures shared between the $300 \mathrm{~V}$ and the 300I grisms.

order-separation filter (OG590) was used with the latter grism. The nebular spectrum of SN 2003hv was obtained using a $1^{\prime \prime} .3$ slit and $2 \times 20$ min exposures per grism. The final wavelength range is 3600-9750 ̊.

\subsection{HST observations}

Late-phase observations in the optical were acquired with HST during two main epochs, at 307 and 433 days past maximum brightness. These data were collected as part of a Snapshot Survey of the sites of Nearby Supernovae ${ }^{2}$. The observations were taken with the Advanced Camera for Surveys (ACS) using the HRC detector and the F435W, F555W, F625W, and F814W filters.

A dedicated $H S T$ program was approved to probe the very late phases of SN $2003 \mathrm{hv}^{3}$. Unfortunately, the optical-band observations, scheduled on day +816 , were never executed due to a technical problem with acquiring a guide star. Deep NIR observations with filter F160W (similar to the $H$-band filter) were obtained with the Near Infrared Camera and Multi-Object Spectrometer (NICMOS) on day +786 . The HST observations are summarized in Table A.3.

\section{Data reductions}

\subsection{Optical photometry}

All images were reduced in a standard manner including bias and flatfield corrections using IRAF scripts ${ }^{4}$. The ground-based photometry of SN $2003 \mathrm{hv}$ was measured differentially with respect to a calibrated sequence of local stars in the field of NGC 1201. Given the wide range in the SN brightness over the different epochs (>12 mag), it was necessary to calibrate both relatively bright and faint local sequence stars. The former were used to measure differential photometry of the SN on the CTIO, KAIT, LCO, and SSO images, while the latter were used with the late-epoch VLT observations. The comparison stars were calibrated with the use of standard-star observations; they are indicated in Fig. 1, and their magnitudes are listed in Table 2. The brightest stars used for the early epochs (i.e., stars 1-7) were calibrated with the help of five sets of

\footnotetext{
2 Program GO-10272, PI Filippenko.

3 Program GO-10513, PI Milne.

${ }^{4}$ IRAF is distributed by the National Optical Astronomy Observatory (NOAO): http://iraf.noao.edu/iraf/web/
}

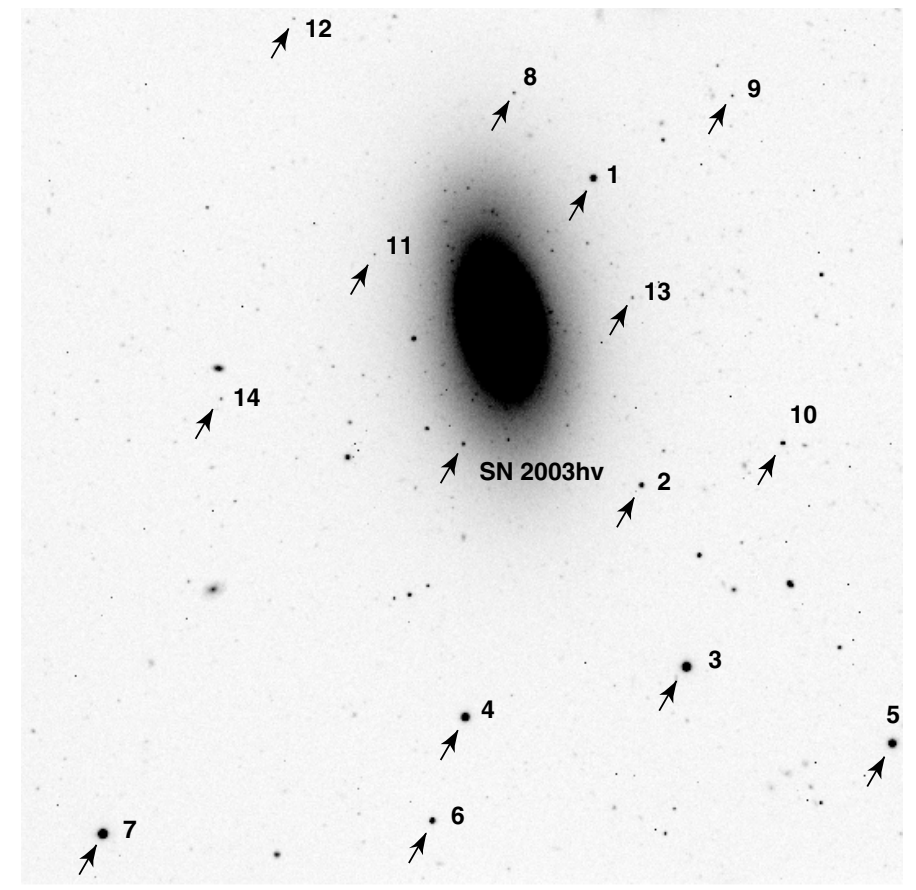

Fig. 1. The galaxy NGC 1201 including SN 2003hv. The field of view is $6.8^{\prime} \times 6.8^{\prime}$; north is up and east to the left. This $B$-band image was obtained on 12 August 2004 with VLT/FORS1, when the SN was almost 340 days past maximum. The local comparison stars are indicated with arrows. Their calibrated magnitudes are listed in Table 2. Stars 1-7 are brighter and were used for differential photometry in images obtained with smaller telescopes, when the SN was bright. Stars 8-14 were used mainly at later times.

observations on three different photometric nights. To calibrate the faint local sequence, the standard-star field PG0231+051 (Landolt 1992) was observed under photometric conditions on 12 August 2004 with the VLT. Instrumental magnitudes of the local stars were measured with a 1'. 6 aperture radius (8 pixels) and then an aperture correction was applied. The associated uncertainties were computed by adding in quadrature the errors associated with the nightly zero-point, the photometry error as computed by the IRAF task phot, and the error associated with the aperture correction.

The host galaxy at the location of the supernova was subtracted from all early-epoch science images (i.e., CTIO, LCO, SSO, KAIT) with the aid of host-galaxy templates. The template 
Table 2. Calibrated magnitudes for the local comparison stars in Fig. $1^{a}$.

\begin{tabular}{cccccc}
\hline \hline Star & $U$ & $B$ & $V$ & $R$ & $I$ \\
\hline 1 & $18.228(0.002)$ & $18.000(0.013)$ & $17.261(0.014)$ & $16.849(0.013)$ & $16.431(0.022)$ \\
2 & $19.712(0.329)$ & $19.254(0.024)$ & $18.071(0.019)$ & $17.323(0.008)$ & $16.644(0.019)$ \\
3 & $17.308(0.009)$ & $16.195(0.009)$ & $14.919(0.010)$ & $14.123(0.010)$ & $13.431(0.008)$ \\
4 & $17.879(0.006)$ & $16.738(0.013)$ & $15.502(0.009)$ & $14.731(0.010)$ & $14.062(0.008)$ \\
5 & $\ldots$ & $16.978(0.012)$ & $16.428(0.016)$ & $16.093(0.016)$ & $15.766(0.005)$ \\
6 & $\ldots$ & $18.612(0.014)$ & $17.779(0.008)$ & $17.253(0.008)$ & $16.797(0.012)$ \\
7 & $\ldots$ & $16.228(0.003)$ & $15.754(0.002)$ & $15.432(0.002)$ & $15.123(0.003)$ \\
8 & $22.179(0.059)$ & $22.029(0.015)$ & $21.290(0.017)$ & $20.847(0.025)$ & $20.470(0.045)$ \\
9 & $21.801(0.058)$ & $21.665(0.011)$ & $20.925(0.016)$ & $20.459(0.023)$ & $20.043(0.042)$ \\
10 & $19.522(0.057)$ & $20.123(0.010)$ & $19.933(0.015)$ & $19.586(0.023)$ & $19.064(0.042)$ \\
11 & $23.595(0.080)$ & $22.479(0.026)$ & $21.008(0.019)$ & $20.069(0.024)$ & $19.069(0.043)$ \\
12 & $23.606(0.071)$ & $22.399(0.014)$ & $20.894(0.016)$ & $19.930(0.023)$ & $19.018(0.042)$ \\
13 & $22.393(0.060)$ & $22.206(0.018)$ & $21.352(0.019)$ & $20.822(0.025)$ & $20.410(0.045)$ \\
14 & $22.384(0.060)$ & $21.850(0.013)$ & $20.885(0.016)$ & $20.302(0.023)$ & $19.834(0.042)$ \\
\hline
\end{tabular}

${ }^{a}$ Numbers in parentheses are uncertainties.

images were obtained with KAIT and the LCO duPont (+Tek5) 2.5-m telescope at times which were sufficiently late that the supernova was no longer detectable. Next, point-spread function (PSF) photometry of the local sequence and the supernova was computed from the template-subtracted images in the manner described by Hamuy et al. (2006). Galaxy subtraction was not performed on the late-time images because the templates were not deep enough. At late times (i.e., VLT), when the SN was faint, aperture photometry with small apertures $\left.(\approx 0 !)^{\prime}\right)$ was used to extract the light from the supernova and the comparison stars. This was done to minimize background contamination from the host galaxy. PSF photometry was also performed (with tasks in the daophot package) and good agreement was found with the aperture-photometry results.

Photometry of the HST images was done following the procedures described in the ACS Data Handbook (Pavlovsky et al. 2006) and in Sirianni et al. (2005). The flux of the supernova was measured directly in the drizzled images with a 7-pixel aperture $(0 . ' 175)$ and converted to the Vega magnitude system using the zero-points provided by the STScI webpage ${ }^{5}$. Aperture corrections as described by Sirianni et al. (2005) and a small charge transfer efficiency (CTE) correction were also applied.

\subsection{S-corrections}

It is well known that combining data obtained with different telescopes can lead to systematic differences in the light curves of SNe. This was the case for SN 2003hv as well, with differences that were most pronounced in the $R$ and $I$ light curves around the time of the secondary $I$-band maximum. To remedy this problem we computed and applied S-corrections to our photometry (Stritzinger et al. 2002). This practice has become a standard procedure in recent years and several authors have obtained encouraging results (see, e.g., Pastorello et al. 2007; Stanishev et al. 2007; Pignata et al. 2008; Wang et al. 2009). In the case of SN 2003hv the number of optical spectra available, especially at phases up to +50 days, does not offer the desired temporal and wavelength coverage necessary to compute accurate S-corrections. For this reason a different approach was chosen and our S-corrections were computed up to day +70 based on the spectral template sequence of Hsiao et al. (2007). At each epoch, the spectral template was multiplied by a smooth spline such that synthetic photometry constructed with this "warped"

5 www.stsci.edu/hst/acs/analysis/zeropoints spectrum matched the observed photometry of SN 2003hv. This is a common practice when using SN spectral templates to compute K-corrections (Nugent et al. 2002; Hsiao et al. 2007).

Armed with the modified template spectra, standard procedures to compute $\mathrm{S}$-corrections were followed; as a reference we adopted the $B V R I$ filter transmission functions from Bessell (1990), modified to account for the photon-counting nature of CCD detectors. The response functions used for KAIT and the CTIO 0.9-m telescope have been published by Wang et al. (2009) and the ones for SSO 2.3-m telescope by Pastorello et al. (2007). We constructed the corresponding response functions for the LCO Swope telescope by multiplying the filter transmission functions with the detector quantum efficiency, the mirror reflectivity, and the atmospheric transmission, as functions of wavelength. To test the accuracy of the modeled passbands, they were used to derive synthetic magnitudes of a set of spectrophotometric standards (Stritzinger et al. 2005). These magnitudes were then used to derive color terms, which were compared to the color terms derived from the broad-band photometry. From this exercise we confirmed that our modeled passbands, in general, reflected a reliable model of the true global response function of each telescope. Finally, in order to compute the S-corrections we convolved the instrumental bandpasses with the spectral template sequence by Hsiao et al. (2007) modified, as described above, to match the SN 2003hv photometry. No corrections were attempted either for the $U$-band, or for epochs past day +70 , since this is where the spectral template sequence of Hsiao et al. (2007) ends.

The resulting values are listed in Table 3 . Due to the fact that these S-corrections were not computed in the optimal way (i.e., based on a well-sampled series of real SN 2003hv spectra), we have chosen to provide the uncorrected photometry of SN 2003hv in Table 4. It is left to any future user of these data to decide whether to make use of these S-corrections. An estimate of their accuracy can be based on the dispersion of the difference between the corrections derived from the templates of Hsiao et al. (2007) and real spectra. In most cases, this error was estimated to be small $(<0.01 \mathrm{mag})$, but in a few cases the uncertainty in the correction was of the same order as the correction itself. This illustrates the limitations of the adopted method. Our experience, however, showed that the S-corrections did reduce the scatter in the light curves, and we have therefore chosen to use them everywhere throughout this paper and recommend their use. Nevertheless, some inconsistencies between the various datasets still remain, especially at times of $+100-140$ days. 
Resolving these remaining discrepancies is beyond the scope of this paper.

\subsection{NIR photometry}

The NIR standards P9106 and P9172 (Persson et al. 1998), were observed with the CTIO $1.3-\mathrm{m}$ telescope on six photometric nights, in order to calibrate field stars in the vicinity of SN 2003hv. $Y$-band magnitudes of the Persson stars were calculated using the following relationship (Krisciunas et al. 2004), derived from synthetic photometry of Vega, Sirius, and the Sun: $\left(Y-K_{\mathrm{s}}\right)=-0.013+1.614\left(J_{\mathrm{s}}-K_{\mathrm{s}}\right)$. Stars 2 and 3 (Fig. 1$)$ were calibrated in this manner; their magnitudes are listed in Table 5. These stars also have $J H K$ values in the Two Micron All Sky Survey (2MASS) and good agreement was found between our values and 2MASS. The NIR photometry of the SN was then calculated differentially with respect to star 3 (the brightest) for all CTIO $1.3-\mathrm{m}$ epochs. Table 6 contains the derived photometry.

Reductions of the VLT NIR images were done with the Eclipse software package (Devillard 1999). The task jitter was used to estimate and remove the sky background from each individual image before creating a stacked image. Photometry of the supernova was determined on the reduced images relative to the two field stars mentioned above and a third 2MASS star in the field of view of ISAAC. Instrumental magnitudes were computed with phot using an aperture with a radius of 0.5 . The quoted uncertainties in Table 6 account for the phot error, the scatter around the zero-point, and the minimum error of the 2MASS sequence. In the cases when two observations were obtained during the same night, they were combined to increase the signal-to-noise ratio. For the final epoch, images from different nights were also combined. However, since there was no detection of the supernova during the final epoch, a $3 \sigma$ upper limit was computed.

For the HST NICMOS data, we used the Mosaic Files (see McLaughlin \& Wiklind 2007) generated by the STScI pipeline. The 16 dithered frames were combined in IRAF. Unfortunately, the SN was located only 5 pixels away from the erratic NICMOS middle column 6 , which proved difficult to correct. For this reason, and the fact that the SN was faint, photometry of the SN was

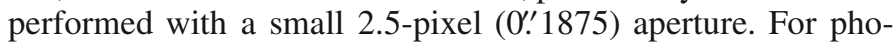
tometry performed on half of the frames (the ones where due to the dithering pattern the $\mathrm{SN}$ was located farther from the bad column), a larger aperture was used and these magnitudes were found to be consistent with the results obtained with the small aperture. The encircled flux was computed using the zero-points provided on the STScI webpage ${ }^{7}$. Appropriate aperture corrections were applied (Barker et al. 2007, Fig. 4.10), and the flux was converted to the Vega-based magnitude system.

\subsection{Spectroscopy}

All spectra were reduced following standard procedures. The two-dimensional frames were bias subtracted and divided by a master flatfield image, and then the cosmic rays were removed. One-dimensional (1-D) spectra were extracted, wavelength calibrated with comparison-lamp spectra, and then flux-calibrated relative to spectrophotometric standard star observations. The wavelength calibration was checked against the night-sky lines, and when appropriate the blue and red spectra were combined

\footnotetext{
6 wwW.stsci.edu/hst/nicmos/performance/anomalies

7 www.stsci.edu/hst/nicmos/performance/photometry
}

to create a final spectrum. Spectra obtained at SSO had telluric features removed with the aid of a telluric standard star.

\section{Results}

We now present the results of our observations, first for the early phases and then for the later epochs. The complete light curves and spectral sequence for SN 2003hv are shown in Figs. 2 and 3 , respectively.

\subsection{Early-phase photometry}

Using the method described by Prieto et al. (2006), we fit template light curves to the early-time S-corrected $B V R I$ photometry. This allowed us to determine basic parameters of the light curves and to estimate the level of host-galaxy reddening. Our slightly modified fitting method is described by Burns et al. (2009). The fits can be seen in the inset of Fig. 2.

The light-curve fit indicates that $B_{\max }$ occurred on (MJD) $=$ $52891.2 \pm 0.3$ (or 9.15 September 2003) with an apparent peak magnitude of $12.45 \pm 0.03$. Peak brightness in the VRI bands occurred at $+0.9,+0.1$, and -2.2 days relative to $B_{\max }$. We note here that the reported uncertainties are the statistical errors of the fit. Since our photometry does not cover the peak, however, a larger systematic uncertainty might be anticipated.

The computed host-galaxy reddening is $E(B-V)_{\text {host }}=$ $-0.04 \pm 0.01 \mathrm{mag}$. Formally, one must also add a systematic uncertainty of $\pm 0.06 \mathrm{mag}$, related to the observed color spread of $\mathrm{SNe}$ Ia. We note that zero reddening is consistent with the position of the supernova in the outskirts of an S0 galaxy.

The light-curve fit of SN $2003 \mathrm{hv}$ yields a $B$-band decline rate $\Delta m_{15}(B)=1.61 \pm 0.02 \mathrm{mag}$. SN 2003hv therefore lies near the faint end of the normal luminosity vs. decline-rate distribution, close to the highly subluminous SN 1991bg-like SNe Ia (e.g., Filippenko et al. 1992), which are observed to have $\Delta m_{15}(B)>1.7 \mathrm{mag}$. To date there is a lack of well-studied SNe Ia with $\Delta m_{15}(B)$ values in the range between 1.5 and 1.7 (see, e.g., Prieto et al. 2006; Mazzali et al. 2007). Currently it is not clear to what extent the properties of normal and subluminous SNe Ia compare. In this sense, SN 2003hv, with its extensive data coverage, may help us understand the similarities or differences between these different types of SNe Ia.

The distance modulus of SN $2003 \mathrm{hv}$ is estimated to be $\mu_{\mathrm{SN}}=$ $31.58 \pm 0.05 \mathrm{mag}$ from these light-curve fits. The quoted error is the statistical error of the fit and we have assumed a Hubble constant of $H_{0}=72 \mathrm{~km} \mathrm{~s}^{-1} \mathrm{Mpc}^{-1}$ (Freedman et al. 2001). There is an additional systematic error (amounting up to $\sim 0.15 \mathrm{mag}$ ) related to the intrinsic dispersion in the luminosity of SNe Ia. This distance is consistent with the SBF distance of NGC 1201 $\mu_{\mathrm{SBF}}=31.37 \pm 0.30 \mathrm{mag}$ (Tonry et al. 2001; Jensen et al. 2003). We conclude that SN 2003hv was a normal-luminosity SN Ia that obeys the Phillips relation (Phillips 1993; Phillips et al. 1999) within its inherent scatter, since this is an underlying assumption for deriving a distance modulus with the aid of the light curves. Throughout the rest of the paper, in order to avoid any circular reasoning, we adopt the $\mu_{\mathrm{SBF}}$ distance to NGC 1201. The conclusion that SN $2003 \mathrm{hv}$ is a normal SN Ia is also supported in part by the existence of a secondary maximum in the I-band, whereas very rapidly declining SN 1991bg-like events lack this feature (Filippenko et al. 1992).

By fitting only the KAIT data, which is a sufficiently large homogeneous dataset, no significant differences were obtained 


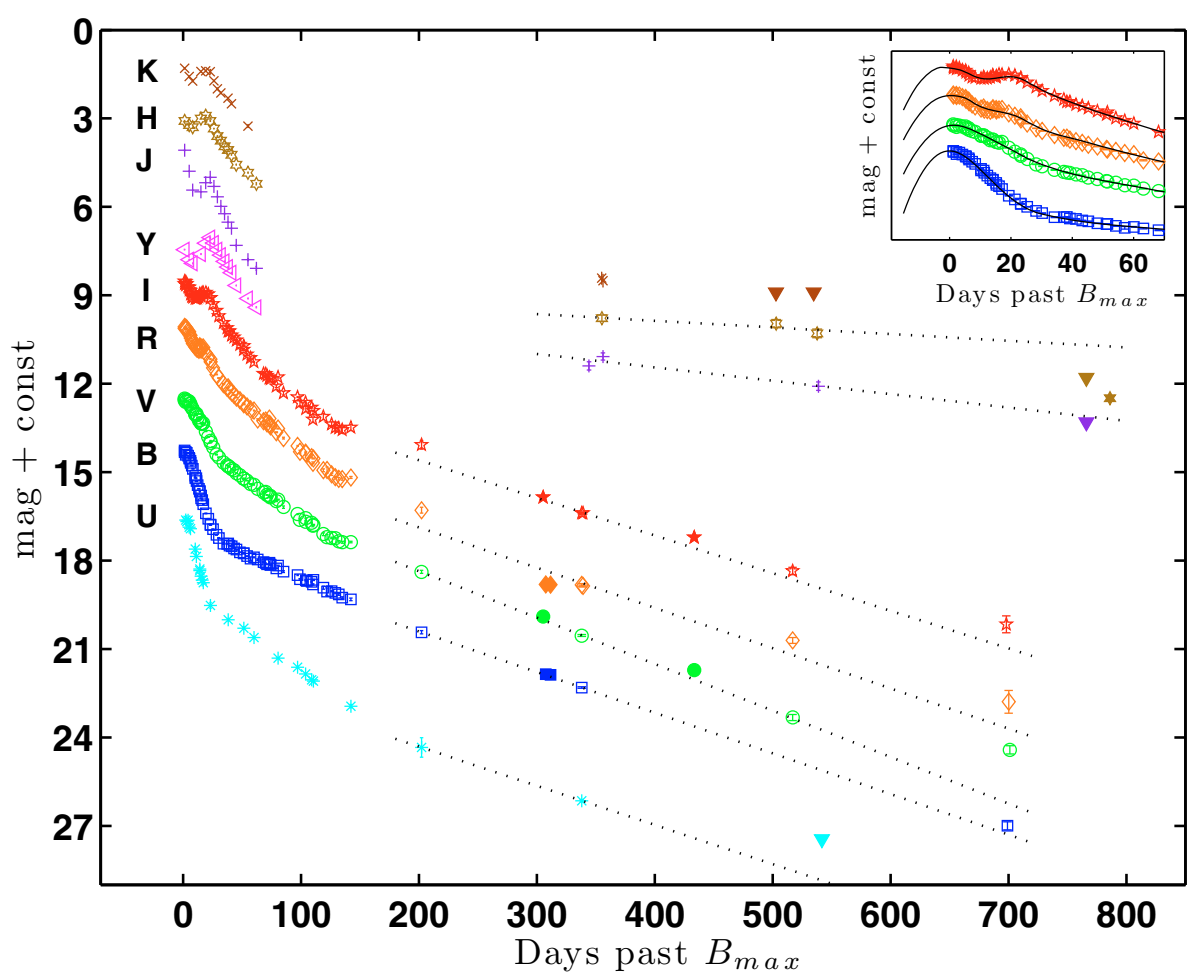

Fig. 2. The UBVRIYJHK light curves of SN 2003hv. They have been shifted for clarity by the following constants: $+4.5,+1.8,0.0$, $-2.4,-4.2,-5.8,-9,-10.2$, and -11.8 , respectively. Filled triangles represent $3 \sigma$ upper limits. The HST points (in the HST filter system) are indicated with filled symbols. Linear fits to the data in the range $+200-540$ days are shown with dotted lines to guide the eye. The inset contains light-curve fits to the early $B V R I$ photometry (Burns et al. 2009); the data have been shifted vertically by different constants than in the main graph.

Table 5. Calibrated NIR magnitudes for local comparison stars in Fig. 1.

\begin{tabular}{ccccc}
\hline \hline Star & $Y^{a}$ & $J$ & $H$ & $K_{s}$ \\
\hline 2 & $16.103(0.017)$ & $15.792(0.011)$ & $15.295(0.011)$ & $14.927(0.031)$ \\
3 & $12.842(0.013)$ & $12.501(0.009)$ & $11.868(0.013)$ & $11.732(0.017)$ \\
\hline
\end{tabular}

${ }^{a}$ There can be an associated systematic uncertainty of 0.03 mag in the $Y$-band calibration (Krisciunas et al. 2004).

for the derived light-curve parameters. However, the scatter around the fit was reduced ( $\chi_{\text {dof }}^{2}$ of 3.9 vs. 9.3 ), since this avoids systematic uncertainties introduced by combining data from different telescopes. The $\chi_{\text {dof }}^{2}$ for the combined dataset without Scorrections was 15.6 .

In Fig. 4, the early observed colors of SN 2003hv are compared with those of the normal SN 1992A (Suntzeff 1996), SN 2001el (Krisciunas et al. 2003), and SN 2003du (Stanishev et al. 2007). These $\mathrm{SNe}$ Ia have $\Delta m_{15}(B)$ values of $1.47,1.15$, and 1.06 , respectively. The $V-R$ and $V-I$ colors of SN 2003hv are similar to those of SN 1992A (the one with the most similar decline rate), while the $B-V$ color starts slightly bluer around maximum (similar to SN 2003du) and then three weeks later matches that of SN 1992A. The $B-V$ color evolution between 30 and 90 days past maximum follows the Phillips/Lira relation (Phillips et al. 1999). Note that in Fig. 4, the colors were only corrected for Galactic extinction to illustrate that SN 2003hv is similar to SNe having little or no host-galaxy reddening (SNe 1992A and $2003 \mathrm{du}$ ). This is additional evidence that SN $2003 \mathrm{hv}$ was a normal SN Ia that suffered negligible host-galaxy extinction.

\subsection{Early-phase spectroscopy}

Figure 3 displays the spectroscopic sequence from 1 to 320 days past maximum. The sequence consists of four early-phase, five "mid-epoch," and one late-phase spectra.

The earliest spectra display intermediate-mass elements characteristic of a normal SN Ia near maximum brightness. The evolution of these spectra confirms that this was a normal event. We have used the "Supernova Identification" code (SNID; Blondin \& Tonry 2007) to compare the early-time spectra of SN 2003hv with a library of supernova templates. SNID indicates a good agreement ( rlap quality parameter values $>10$ ) with several normal SNe Ia (e.g., SNe 1992A, 1994D, and 1996X) at epochs similar to those deduced from our light-curve fits, to within a few days.

At maximum light, the ratio of the depth of the Si II $\lambda 5972$ and $\lambda 6355$ absorption features was $0.40 \pm 0.05$. This value is somewhat larger than what is measured in other normal SNe Ia (SN 1992A being the runner-up with 0.38), but smaller than values found for subluminous SN 1991bg-like events (Nugent et al. 1995; Garnavich et al. 2004; Benetti et al. 2005).

From the four early-epoch spectra, the rate of decrease in the expansion velocity of the Si II $\lambda 6355$ feature was measured to be $\dot{v}=41 \pm 6 \mathrm{~km} \mathrm{~s}^{-1} \mathrm{day}^{-1}$. By comparing with a large sample of events, we find that SN 2003hv lies in the low-velocity gradient (LVG) group as defined by Benetti et al. (2005).

\subsection{Late-phase photometry}

The measured decay rates of the late-time light curves are listed in Table 7. To facilitate comparison with results of previous studies (Lair et al. 2006; S04; SS07), these decline rates were computed as linear slopes at time intervals out to $\sim 540$ days. The measured late-phase $B V R$-band decline rate of $\sim 1.4$ mag per 100 days in SN $2003 \mathrm{hv}$ is similar to values reported for other well-studied SNe Ia. A possible exception is the I-band, which 
Table 6. NIR photometry of SN $2003 \mathrm{hv}$.

\begin{tabular}{|c|c|c|c|c|c|c|c|}
\hline $\begin{array}{l}\text { Date } \\
\text { (UT) }\end{array}$ & $\begin{array}{l}\text { MJD }^{a} \\
\text { (days) }\end{array}$ & $\begin{array}{l}\text { Phase } \\
\text { (days) }\end{array}$ & Telescope & $\begin{array}{c}Y \\
(\mathrm{mag})\end{array}$ & $\begin{array}{c}J^{b} \\
(\mathrm{mag})\end{array}$ & $\begin{array}{c}H \\
(\mathrm{mag})\end{array}$ & $\begin{array}{c}K_{s} \\
(\mathrm{mag})\end{array}$ \\
\hline 20030910 & 52892.37 & 1.2 & CTIO $1.3 \mathrm{~m}$ & $13.252(0.015)$ & $13.081(0.015)$ & $13.286(0.015)$ & $13.100(0.018)$ \\
\hline 20030914 & 52896.28 & 5.1 & CTIO $1.3 \mathrm{~m}$ & $13.591(0.015)$ & $13.792(0.015)$ & $13.423(0.015)$ & $13.378(0.021)$ \\
\hline 20030917 & 52899.28 & 8.1 & CTIO $1.3 \mathrm{~m}$ & $13.716(0.015)$ & $14.435(0.015)$ & $13.502(0.015)$ & $13.529(0.026)$ \\
\hline 20030924 & 52906.31 & 15.1 & CTIO $1.3 \mathrm{~m}$ & $13.403(0.015)$ & $14.491(0.015)$ & $13.202(0.015)$ & $13.211(0.018)$ \\
\hline 20030928 & 52910.26 & 19.1 & CTIO $1.3 \mathrm{~m}$ & $13.031(0.015)$ & $14.179(0.015)$ & $13.112(0.015)$ & $13.189(0.022)$ \\
\hline 20031002 & 52914.20 & 23.0 & CTIO $1.3 \mathrm{~m}$ & $12.845(0.016)$ & $14.000(0.018)$ & $13.283(0.020)$ & $13.216(0.073)$ \\
\hline 20031005 & 52917.25 & 26.1 & CTIO $1.3 \mathrm{~m}$ & $13.025(0.015)$ & $14.306(0.015)$ & $13.546(0.015)$ & $13.524(0.020)$ \\
\hline 20031008 & 52920.24 & 29.0 & CTIO $1.3 \mathrm{~m}$ & $13.255(0.015)$ & $14.657(0.015)$ & $13.799(0.015)$ & $13.796(0.026)$ \\
\hline 20031011 & 52923.22 & 32.0 & CTIO & $13.439(0$. & $14.980(0$. & $13.958(0$. & $13.936(0.022)$ \\
\hline 20031014 & 52926.25 & 35.1 & CTIO & $13.650(0$ & 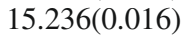 & $14.145(0$. & \\
\hline 20031017 & 52929.22 & 38.0 & CTIO & 13.8 & 15. & $14.273(0$. & $14.116(0.026)$ \\
\hline 20031 & 2.17 & 41.0 & CTIC & 14. & 15. & $14.431(($ & $14.306(0.024)$ \\
\hline 20031024 & 52936.21 & 45.0 & CTIO & 14. & 16.310( & $14.770(C$ & \\
\hline 20031103 & 52946.21 & 55.0 & CTIC & $14.912(($ & 16.790( ( & $15.041(0$ & $15.060(0.048)$ \\
\hline 20031110 & 52953.22 & 62.0 & CTIO $1.3 \mathrm{~m}$ & $15.207(0.017)$ & $17.088(0.0$ & $15.420(0.037)$ & \\
\hline 20040818 & 53235.37 & 344.2 & VLT A & .. & $20.394(0.140)$ & & \\
\hline 20040829 & 53246.31 & 355.1 & VLT A & $\ldots$ & & $19.977(0.106)$ & $20.221(0.216)$ \\
\hline 20040830 & 53247.28 & 356.1 & VLT Antu & .. & $20.078(0.122)$ & & $20.303(0.224)$ \\
\hline 20050124 & 53394.06 & 502.9 & VLT Antu & ... $>>>>$ & 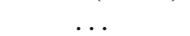 & $20.163(0.138)$ & $>20.700$ \\
\hline 20050224 & 53425.99 & 534.8 & VLT Antu & .. $>$ & 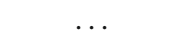 & & $>20.700$ \\
\hline 20050227 & 53428.99 & 537.8 & VLT Antu & $\ldots$ & .. & $20.495(0.146)$ & $\cdots$ \\
\hline 20050228 & 53429.99 & 538.8 & VLT Antu & .. & $21.087(0.144)$ & & \\
\hline $20051014^{c}$ & 53657.07 & 765.9 & VLT Antu & .. & $>22.310$ & $>22.000$ & \\
\hline 20051103 & 53677.31 & 786.1 & $H S T$ NICMOS $^{d}$ & .. & .. & $22.691(0.051)$ & \\
\hline
\end{tabular}

${ }^{a}$ All images obtained the same night are referred to a mean MJD.

${ }^{b} \mathrm{~A} J$ filter was used at the CTIO $1.3 \mathrm{~m}$ telescope, while $J_{s}$ was used at the VLT.

${ }^{c}$ All images obtained on October 13-15 are averaged here.

${ }^{d}$ Vega magnitudes in the HST filter system $(F 160 W)$.

is found to decline slower than $B V R$, but not as slowly as found in other $\mathrm{SNe}$ Ia (i.e., $~ 1.0 \mathrm{mag}$ per 100 days). In addition, as discussed by SS07, the $U$-band has a late-time decline rate comparable to that of $B V R$ for at least up to 340 days.

However, in the case of SN 2003hv, the evolution of the lateepoch light curve is, for the first time, followed in multiple bands out to 700 days past maximum light. At these very late stages, an apparent slowdown of the optical light curves is observed; the drop in luminosity appears to be decelerating. This behavior is most pronounced in the $V$-band, where it has also been observed in several other SNe Ia. We discuss this further in Sect. 5.3.

In the NIR bands, SN 2003hv displays a different behavior than the constant brightness observed during late-phase observations of SNe 2000cx and 2001el (S04 and SS07, respectively). SN $2003 \mathrm{hv}$ slowly fades in brightness between days +350 and +540 . Furthermore, the $\mathrm{SN}$ is not detected in the VLT images at +766 days, indicating a further drop during this period. However, we do detect a point-like source in the HST/NICMOS/F160W image obtained at +786 days (see Fig. 5). From astrometry relative to nearby stars with the help of the deep VLT $H$-band images, we establish that this object's position is consistent with the position of the supernova to within $0{ }^{\prime} 061 \pm 0 .{ }^{\prime} 085$ (where the quoted error is the transformation error). With a magnitude of $22.69 \pm 0.05$, this is to our knowledge the latest detection ever achieved of a SN Ia in the NIR.

Notice that the HST ACS observations were not included in the linear fits in Table 7. This is because the HST and groundbased filter systems are significantly different. These differences can produce systematic effects in the photometry, especially when one considers the nonstellar nature of the spectral energy distribution of SNe Ia. Synthetic photometry computed with the nebular spectrum and the VLT and HST filter transmission functions indicates that on day +320 the $\mathrm{SN}$ would appear brighter by $0.20,0.25$, and $0.28 \mathrm{mag}$ in $B V I$ and fainter by 0.37 mag in $R$ in the HST filter system ${ }^{8}$. However, the farther we move from the epoch of the nebular spectrum, the less accurate these corrections become; hence, they were not applied. Nevertheless, the ACS points lie fairly close to the calculated linear slopes.

The $R$-band +516 day observation was obtained using the FORS2 "special" $R$-band filter, which differs from the Bessell $R$ filter. Synthetic photometry computed with the nebular spectrum reveals that SN $2003 \mathrm{hv}$ would appear $\sim 0.08$ mag brighter in the $R_{\text {special }}$ filter at +320 days. Assuming that the shape of the spectrum does not change out to +516 days, this induces a change to the respective $R$-band decline rates by no more than half a standard deviation, and it has no consequence to the following discussion.

\subsection{Late-phase spectroscopy}

The nebular spectra (bottom Fig. 3) of SN 2003hv are, generally speaking, similar to those of other normal $\mathrm{SNe}$ Ia, and display several broad iron-group emission features. In particular, the latest spectrum is dominated by strong Fe emission features at $\sim 4700 \AA$ and $5250 \AA$. These are produced from the blending of forbidden transitions associated with Fe II and Fe III.

Motohara et al. (2006) and Gerardy et al. (2007) found in the NIR and MIR nebular spectra of SN 2003hv that the [Fe II] $1.644 \mu \mathrm{m}$ and [Co III] $11.89 \mu \mathrm{m}$ emission lines were blueshifted by $\sim 2600 \mathrm{~km} \mathrm{~s}^{-1}$. These shifts were interpreted as the result of an asymmetric, off-centre explosion. In addition, the [Fe II] $1.644 \mu \mathrm{m}$ line had a flat-topped profile. It was therefore argued

\footnotetext{
${ }^{8}$ The F625W is actually an $r$, not an $R$, filter.
} 
Table 7. Decline rates in mag per 100 days.

\begin{tabular}{lcccccccc}
\hline \hline Epoch & $U$ & $B$ & $V$ & $R$ & $I$ & $J_{s}$ & $H$ & $K_{s}$ \\
\hline $200-540$ days $^{a}$ & $1.33(0.24)$ & $1.38(0.05)$ & $1.58(0.03)$ & $1.37(0.05)$ & $1.28(0.06)$ & $0.45(0.09)$ & $0.23(0.09)$ & $>0.30$ \\
\hline
\end{tabular}

${ }^{a}$ In the $U$-band only to 340 days.

${ }^{b}$ Numbers in brackets are the formal errors of the weighted least-squares linear fits to the photometric data.

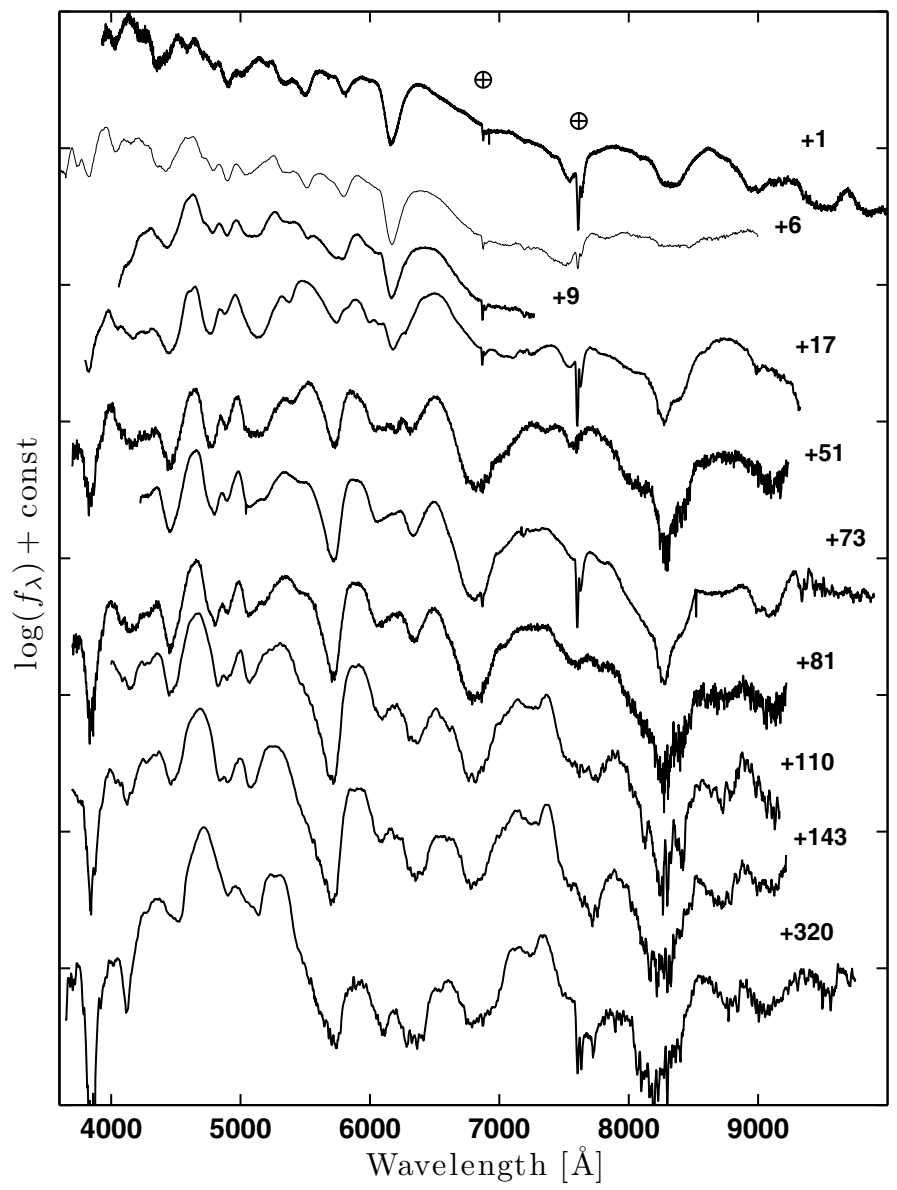

Fig. 3. Spectral evolution of SN 2003hv from maximum light to the nebular phase. Numbers indicate days past maximum brightness. For clarity, the spectra have been offset in flux scale with respect to each other. The Earth symbols mark telluric features. Note that the SSO spectra have had their telluric lines removed. The bottom three spectra have been smoothed (by a moving average of 5 pixels) for presentation purposes.

(Motohara et al. 2006) that regions below $\sim 3000 \mathrm{~km} \mathrm{~s}^{-1}$ are filled with neutron-rich non-radioactive isotopes (see also Höflich et al. 2004).

We examined our optical nebular spectrum to investigate whether any signatures of blueshifted lines or flat-topped profiles were present. It should be stressed that the optical spectrum is produced from the blend of many overlapping transitions, and it is therefore problematic to make strong claims from the appearance of optical lines. However, a fairly isolated [Fe II] feature is present at $8621 \AA$. This line, which often falls outside the wavelength coverage of optical spectra, contributes according to our modeling (see Sect. 5.2) $90 \%$ of the flux in this wavelength region. This is comparable to the "cleanness" of the [Fe II] $1.644 \mu \mathrm{m}$ line in the NIR and higher than the $65 \%$ contribution that our model suggests for the [Fe II] $\lambda 7155$ line in its corresponding region. The latter line, which is contaminated mainly
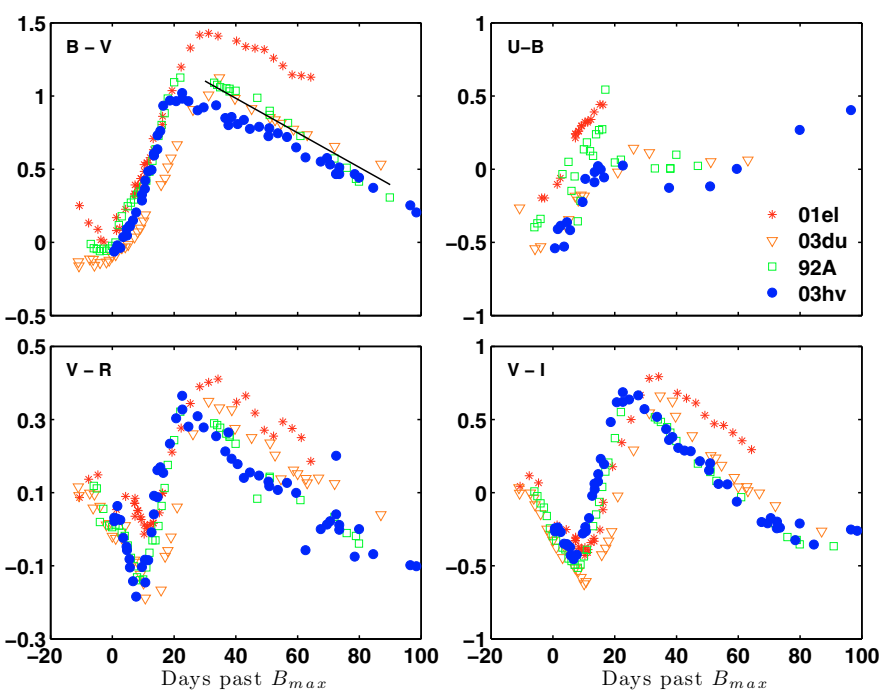

Fig. 4. Color evolution of SN 2003hv from maximum light to 100 days past maximum. For comparison, we have plotted the colors of SN 1992A (Suntzeff 1996), SN 2001el (Krisciunas et al. 2003), and SN 2003du (Stanishev et al. 2007). The Phillips/Lira relation (Phillips et al. 1999) is also indicated in the $B-V$ panel (solid line). The four SNe have only been corrected for Galactic reddening $(E(B-V)=0.016$, $0.017,0.014$, and $0.010 \mathrm{mag}$, respectively, Schlegel et al. 1998). This illustrates that SN 2003hv, as in the cases of SNe 1992A and 2003du, suffers little to no extinction from its host. SN 2001el, on the other hand, was substantially reddened and clearly does not follow the Phillips/Lira relation.

by [Ca II], was inspected by Höflich et al. (2004) in the case of SN 2003du, and was found to have a peak "seemingly in contradiction" with the boxy NIR profiles. In Fig. 6 the $8621 \AA$ and $1.644 \mu \mathrm{m}$ (Motohara et al. 2006) lines are compared in velocity space. We see that these two features have somewhat similar profiles and both are apparently blueshifted by $\sim 2600 \mathrm{~km} \mathrm{~s}^{-1}$. The same could be argued for the $7155 \AA$ line. Other iron lines are more heavily blended and definitely unsuitable for such diagnostics (notice, however, that the $5250 \AA$ feature is also flat-topped). This could be considered as supporting the findings presented by Motohara et al. (2006) and Gerardy et al. (2007). However, in addition to the concerns raised above, these lines do show some evolution (Fig. 3), which could be additional evidence pointing toward blending.

\section{Discussion}

\subsection{SN 2003hv in the context of its $\Delta m_{15}(B)$ value}

In this subsection, the properties of SN 2003hv are reviewed in the light of its rather uncommon light-curve decline-rate parameter. There is a general division between normal and subluminous $\mathrm{SNe}$ Ia, where the latter are typically observed to have $\Delta m_{15}(B)>1.7 \mathrm{mag}$. The subluminous $\mathrm{SNe}$ Ia do not seem to follow the linear luminosity vs. decline-rate relation of normal SNe Ia (Phillips 1993; Prieto et al. 2006), although an 

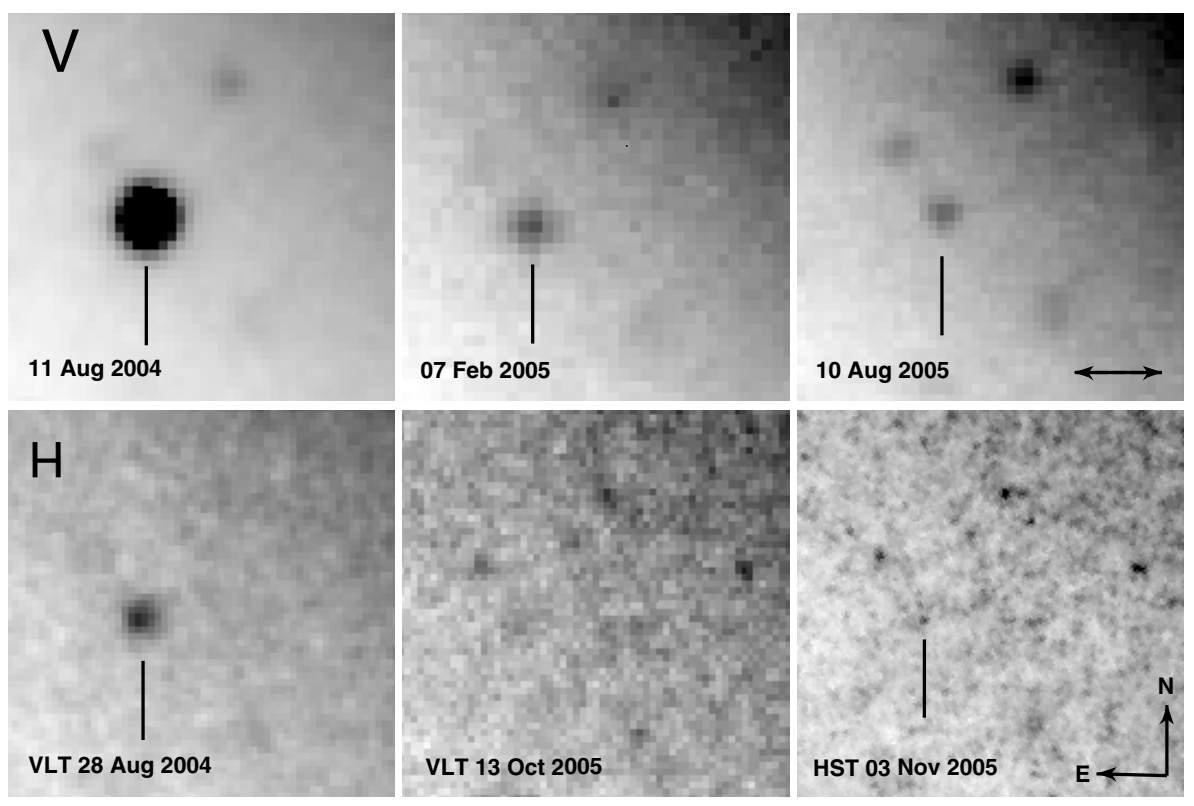

Fig. 5. The fading of SN $2003 \mathrm{hv}$ between days +340 and +786 as observed in the $V$-band (upper sequence) and the $H$-band (lower sequence). The double arrow in the last $V$-band frame is $2^{\prime \prime}$ across. SN 2003hv is clearly detected in all $V$-band images. It is not significantly detected in the VLT/ISAAC $H$-band image from 13 October 2005, at an upper limit of 22 mag. However, SN 2003hv is detected in a deeper image obtained 3 weeks later with HST/NICMOS on 786 days past maximum with a magnitude of $22.69 \pm 0.05$. This is the latest-ever detection of a SN Ia in the NIR. The last image is constructed from the drizzled combination of 16 frames.

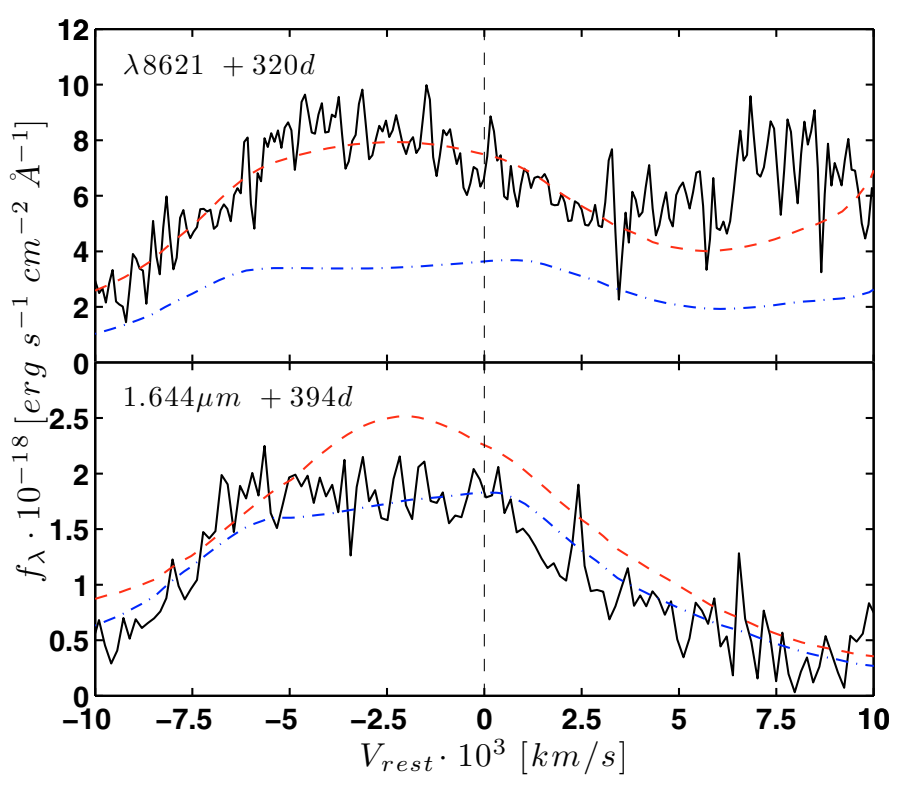

Fig. 6. Comparison of the [Fe II] line profiles at $8621 \AA$ (our spectrum) and $1.644 \mu \mathrm{m}$ (Motohara et al. 2006) in velocity space. These are relatively clean, isolated [Fe II] lines according to our model. The two lines appear equally blueshifted, and their blueshift is equal to the blueshift of [Co III] $11.89 \mu \mathrm{m}$ (see Motohara et al. 2006; Gerardy et al. 2007). The $1.644 \mu \mathrm{m}$ line exhibits a flat-topped profile while the $8621 \AA$ line is not clearly "peaked" (assuming it is not a result of blending by neighboring lines). Also shown are the model spectra (without photoionization) at +300 days (red dashed line) and at +400 days (blue dashed-dotted line) blueshifted by $2600 \mathrm{~km} \mathrm{~s}^{-1}$. In the top panel, their flux has been scaled down by a factor of 7.5 so that the +300 -day model matches approximately the observed flux. The same has been done in the bottom panel, matching the +400 -day model by scaling it down by a factor of 10 . Notice that the $8621 \AA$ line is also expected to develop a clear flat-topped profile at later phases.

exponential fit might be able to include them (e.g., Garnavich et al. 2004). It is not clear whether subluminous SNe Ia can be related to a different class of progenitors or explosion mechanisms (see, e.g., Hillebrandt \& Niemeyer 2000, and references therein).
As very few SNe Ia with $1.5<\Delta m_{15}(B)<1.7$ have been observed (and none in as much detail as SN 2003hv), we feel it is warranted to discuss SN 2003hv in this context, even if it is just a single example, and confirm that it is a "normal" member of the SN Ia family. In particular, with respect to several relations proposed for $\mathrm{SNe}$ Ia, we make the following remarks.

- Adopting the independent SBF distance measurement, an absolute magnitude of $B_{\max }=-18.99 \pm 0.35$ is deduced for SN $2003 \mathrm{hv}$, which is fully consistent with the expected luminosity from its decline rate (linear fits, by Prieto et al. 2006, give -19.0).

- Adding the SN $2003 \mathrm{hv} \mathcal{R}(\mathrm{Si}$ II) value $(0.40 \pm 0.05)$ to the $\mathcal{R}\left(\mathrm{Si}\right.$ II) vs. $\Delta m_{15}(B)$ correlation (Garnavich et al. 2004; Benetti et al. 2005), we find that this data point nicely connects the normal $\mathrm{SNe}$ Ia to the subluminous group in a previously unexplored area of this relation.

- Mazzali et al. (1998) showed that the full width at halfmaximum intensity (FWHM) of the line at $4700 \AA$ in nebular spectra of SNe Ia correlates well with $\Delta m_{15}(B)$. Although SN 2003hv has a FWHM value slightly larger than the one expected by the correlation in Mazzali et al. (1998), it is consistent with the existence of such a correlation.

- Milne et al. (2001) suggested the cut between the normal and subluminous SNe Ia to occur at $\Delta m_{15}=1.6 \mathrm{mag}$ as far as the late light-curve shape is concerned. However, this was based on very few $\mathrm{SNe}$ in the appropriate range and the study was later updated with the inclusion of SN 1999by (Milne $\&$ Williams 2005). We note here that in this sense the latetime light-curve evolution of SN $2003 \mathrm{hv}$ is intermediate between SN 1992A and the transitional (with respect to latetime light-curve behavior) SN 1986G.

- The peak luminosity of SN 2003hv in the NIR bands is comparable to those of normal SNe Ia. As pointed out by Krisciunas et al. (2009), this is also the case for a sub-sample of SNe Ia with $\Delta m_{15}(B)>1.6$, that peak in the $J$-band before the $B$-band. Although our observations do not seem to cover the $J H K$ peaks, this is almost certainly the case for SN $2003 \mathrm{hv}$ and estimates based on the templates by Krisciunas et al. (2004) give $-18.52,-18.17$, and $-18.33( \pm 0.31)$ for $M_{J}$, $M_{H}$, and $M_{K}$, respectively. The assertion of normal peak 
brightness does not change even if the observed maxima are used as lower limits, instead of extrapolating back in time.

- We would not expect to see a SN with such a $\Delta m_{15}(B)$ in a late-type galaxy (Hamuy et al. 2000; Jha et al. 2007). Indeed, its presence in an S0 galaxy is not surprising.

The main conclusion is that SN 2003hv appears to obey many of the known correlations with respect to the $B$-band decline-rate relation and is an object that is similar to the other normal SNe Ia used to derive these correlations. Seen from another perspective, we show that these particular correlations also hold for this previously underexplored value of $\Delta m_{15}(B)$.

\subsection{Nebular spectrum synthesis}

A unique aspect of SN 2003hv is our broad wavelength coverage at nebular phases, when including the IR spectra from Motohara et al. (2006) and Gerardy et al. (2007). This facilitates comparison with model spectra in an unprecedented manner. We have used our detailed spectral synthesis code (described in detail by Kozma \& Fransson 1998a,b; Kozma et al. 2005; S04) to generate a nebular spectrum of SN 2003hv and compare it with the observational data. The spectral synthesis code includes a selfconsistent ionization and level population model to calculate the emission from each radial zone of the ejecta. Nonthermal excitation and ionization by gamma rays and positrons are included, as well as time-dependent effects. Nonlocal scattering of the emission, however, is not included. The present calculations use the W7 hydrodynamical model (Nomoto et al. 1984) as input.

Since the nebular spectra of SN $2003 \mathrm{hv}$ were all obtained at slightly different epochs, the optical and NIR spectra were scaled in flux with the aid of our photometry to match the date of the MIR spectrum. The $V$ and $H$ magnitudes were interpolated to day +358 , assuming the linear slopes from Table 7 . The final combined spectrum is shown in Fig. 7 together with our model spectrum at day +400 .

The synthetic spectrum does a reasonable job in reproducing the general features of the observed spectrum. The dominant $\mathrm{Fe}$ peaks in the range 4000-5500 $\AA$ are reproduced accurately, as is the shape of the spectrum at 7000-9000 $\AA$. In the NIR, the relative fluxes of the $[\mathrm{Fe} \mathrm{II}]$ lines agree with the observations, but the absolute fluxes are overpredicted. In the MIR, our model instead underpredicts the flux levels. The model also predicts a strong stable (non-radioactive) [Ni II] line at $6.64 \mu \mathrm{m}$, which is also seen in the observed spectrum. [Ar II] and [Co II] lines are identified, as mentioned by Gerardy et al. (2007). Note that our modeling includes two different ways to treat the effect of the scattering of UV photons and their subsequent consequences for the photoionization of the metals (representing two limiting cases): with and without photoionization (see, e.g., S04). Here only the model without photoionization (i.e., assuming that all UV photons are redistributed to longer wavelengths in the scattering process) has been plotted because it is a better fit to the observed spectrum. The model with photoionization is not able to reproduce the bluest peak around the $4000 \AA$ bumps, since the relevant $[\mathrm{Fe} \mathrm{I}]$ emission is suppressed in this case.

An interesting feature, as mentioned above, is the flat-topped profiles observed in the NIR lines. It has been suggested that a detailed study of the [Fe II] $1.644 \mu \mathrm{m}$ NIR feature could be the cleanest probe of the ejecta kinematics (e.g., Höflich et al. 2004, and references therein). Motohara et al. (2006) discussed that the NIR nebular spectra of two out of four SNe Ia exhibit a flattopped profile in this line, with SN 2003hv being the strongest case and the one that has been observed farthest from maximum brightness. Our model was first used to investigate how "clean" this line is. It was confirmed that even though the nebular spectrum consists of a large number of overlapping lines, within our model Fe II appears to dominate the $1.7 \mu \mathrm{m}$ region. There are a number of different Fe II transitions that contribute to the feature, but since predominantly recombination radiation is seen at this phase, uncertainties in the ionization are less important in modeling the feature. As pointed out above, we also propose that the [Fe II] $\lambda 8621$ line is relatively clean and isolated from other contributing lines.

The scenario favored by Motohara et al. (2006) and Gerardy et al. (2007) to explain the flat-topped line profiles is an inner "hole" of unmixed, neutron rich, non-radioactive, iron-group elements in the core. These are the products of electron capture, which takes place in the highest density burning regions. Such a configuration of chemical elements is observed in 1-D deflagration explosion models (such as W7), or even 1-D delayed detonation (DDT) models, but is incompatible with current 3D deflagration models, which predict much mixing (e.g., Röpke 2005; Röpke et al. 2007). Motohara et al. (2006) modeled the line and found a slight asymmetry due to overlapping [Fe II] 1.664 and $1.667 \mu \mathrm{m}$ lines, but argued that the overall flatness nevertheless suggested no efficient mixing between the highest density burned region and its surroundings.

Our modeling of the optical-IR spectrum at +320 days, contemporaneous with the optical spectrum, did not show any evidence for flat-topped line profiles. However, allowing the model to run to even later phases, the flat-topped line profiles start to develop. By 400 days past maximum, the line becomes flat-topped (Fig. 6), as also noted by Motohara et al. (2006). Note that our model assumes complete and in situ deposition of the energy carried by the positrons. The core of the ejecta is the densest region where both gamma rays and positrons are most efficiently deposited. The evolution from peaked to flat-topped can thus be explained by the fact that even at +300 days, there is still a fair fraction of energy deposited by gamma rays, and we can follow these as they are deposited in the central region. At even later epochs, however, the energy is provided solely by the positrons. Since these are not able to penetrate to the central regions, the absence of radioactive material in the center will give rise to a flattopped line profile. In this sense, the flat-topped line is consistent with complete and local positron trapping, providing a diagnostic complementary to the late-time light curves (see Sect. 5.5).

Our optical spectrum is useful for constraining some of the alternative explanations that have been proposed for the flattopped line profile. The alternative idea that the profile is due to a dusty core (Motohara et al. 2006) can be ruled out because no such evidence is seen at optical wavelengths. Furthermore, the possibility mentioned by Gerardy et al. (2007), that the hole may be filled with a large fraction of unburned material at low velocities as suggested by some 3-D deflagration models (e.g., Röpke 2005), is also very unlikely: the absence of the [O I] emission at $6300 \AA$ is clearly inconsistent with such a scenario, as discussed by Kozma et al. (2005). Gerardy et al. (2007) further mention, but do not favor, the interaction of the companion star as a possible cause of the hole. This may indeed be interesting to investigate in light of the new models by Pakmor et al. (2008), suggesting the presence of a hole in the wake of the explosion, but the observable signatures of such a hole filled by the companion star are still rather unclear.

Finally, an alternative explanation, proposed here, for the lack of emission below $2500 \mathrm{~km} \mathrm{~s}^{-1}$, could be that an IRC has taken place in the highest density regions. This scenario is further discussed in Sect. 5.6. 


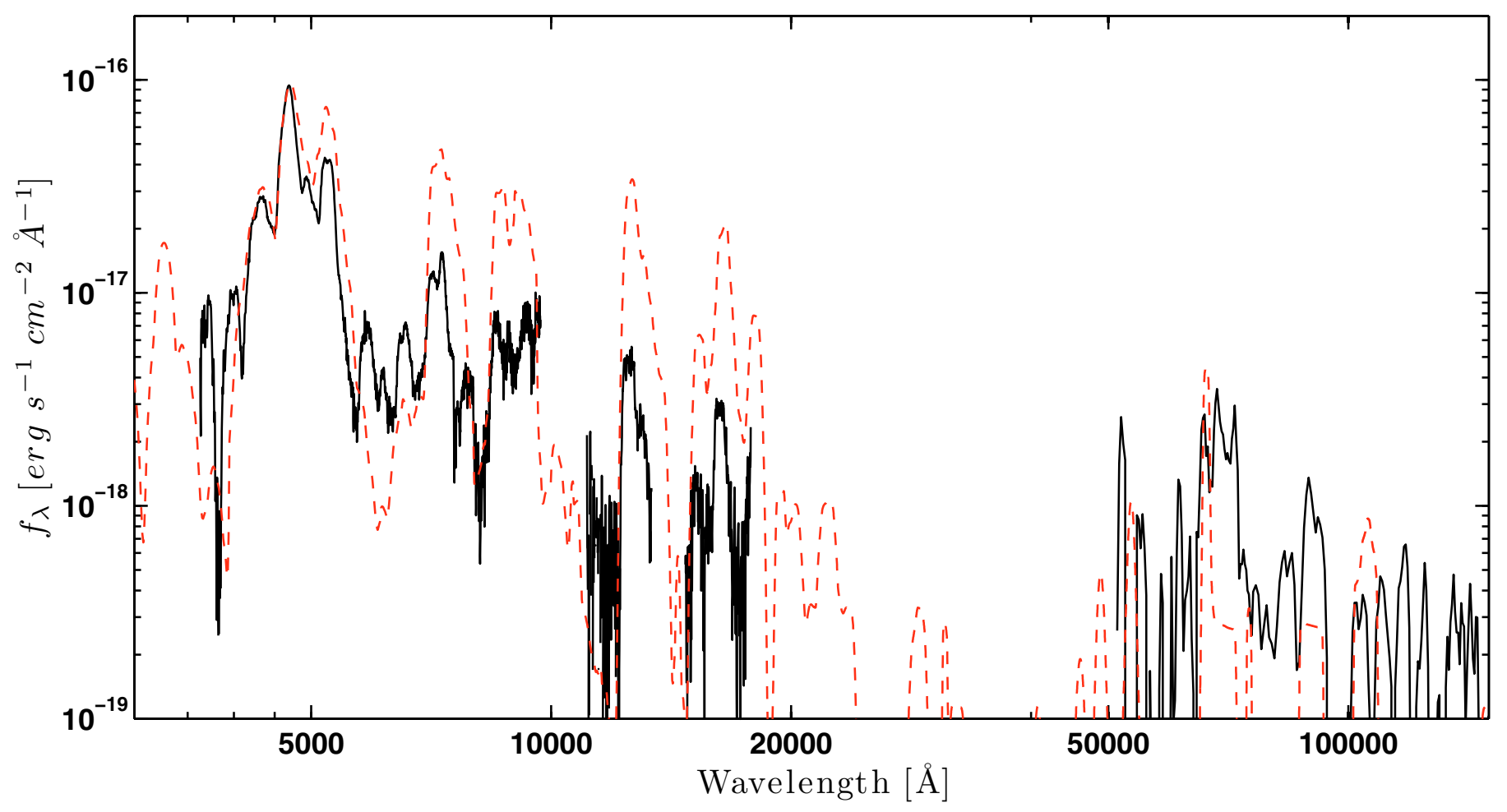

Fig. 7. The multi-wavelength nebular spectrum of SN 2003hv (solid line). This spectrum is a compilation of our optical spectrum at +320 days, the NIR spectrum of Motohara et al. (2006) at +394 days, and the Spitzer MIR spectrum of Gerardy et al. (2007) at +358 days. The optical and NIR spectra have had their flux scaled to match the age of the MIR spectrum with the aid of the $V$-band and $H$-band photometry (see text). For presentation purposes each spectrum was smoothed by a moving average of 3 pixels. Note also that the MIR spectrum has large associated error bars that are not shown here. The dashed red line shows our model spectrum (without photoionization) at +400 days.

\subsection{Slowdown of optical decay}

At very late times, the optical decline rates of SN 2003hv appear to be slowing down, especially in the $V$-band. We first examine whether our photometry could be contaminated by a light echo or a background (or foreground) source. Light echoes have been observed in the past for a handful of SNe Ia (Schmidt et al. 1994; Cappellaro et al. 2001; Crotts \& Yourdon 2008; Wang et al. 2008). However, no evidence of extended structure was observed in our PSF subtractions, neither in the final epoch nor in the highresolution HST images at +430 days. On the other hand, not all possible echo geometries can be resolved at this distance. In the single-scattering approximation (Patat 2005), it is estimated that only echos in intervening clouds at distances greater than $\sim 75 \mathrm{pc}$ from the SN would be resolved by ACS. But in addition, our nebular spectrum shows no evidence of blue continuum and the final colors are not particularly blue. SN $2003 \mathrm{hv}$ did not explode in a star-forming region, but in the outskirts of an S0 galaxy where no dust is expected and there are no signs of host extinction. Finally, if the latest-epoch photometry were dominated by a coincident background source, the corrected slope at 200-500 days would become steeper than those previously observed for SNe Ia at these phases, indicating that this is probably not the case.

We therefore believe that the final photometry indeed measures the supernova light. Actually, such a slowdown has been previously seen in several other SNe Ia observed beyond 600 days past maximum in single (usually $V$-band) observations: SN 1992A (Cappellaro et al. 1997), SN 2000E (Lair et al. 2006), and SN 2000cx (S04, their Sect. 6.5). Many of the same considerations apply in the case of SN 2003hv. However, our multicolor observations suggest that this slowdown may not be characteristic of only the $V$-band. This questions the speculations in
S04 about [Fe I] predominantly emitting in the $V$-band being the explanation for this behavior.

The main consequence of this observation is that the dramatic IRC predicted by some models did not occur at these phases.

\subsection{Bolometric light curve and ${ }^{56} \mathrm{Ni}$ mass}

To construct an UV-optical-NIR (UVOIR) light curve of SN 2003hv, we used the UBVRIJHK photometry from Tables 4 and 6 , including the $\mathrm{S}$-corrections from Table 3 . For missing epochs, the photometry was interpolated by fitting suitable functions to the data. At early times, spline interpolation was used, while at the late nebular phases, linear fits were initially assumed. However, in some bands, due to deviations from the linear decay, quadratic or cubic polynomials gave better fits to the data and were adopted.

In the case of the $U$-band, it was assumed that the light curve continued the linear decay obtained out to +340 days. For the $K$ band we made the limiting assumption that it was barely not detected at +534 days, while the further assumption was made that the $J-H$ and $H-K$ colors do not change between +530 and +786 days, in order to estimate $J$ and $K$ magnitudes at the final epoch. None of the upper limits in $U J H K$ bands was violated by any of these assumptions or fits. The photometry was subsequently corrected for Galactic extinction assuming $R_{V}=3.1$ and following the prescription of Schlegel et al. (1998). Magnitudes were converted to fluxes within the individual filters and the UVOIR flux (and its associated error) was obtained by integrating the filter fluxes over wavelength. The UVOIR luminosity was calculated assuming a distance of $18.79 \pm 2.60 \mathrm{Mpc}$. No corrections were 


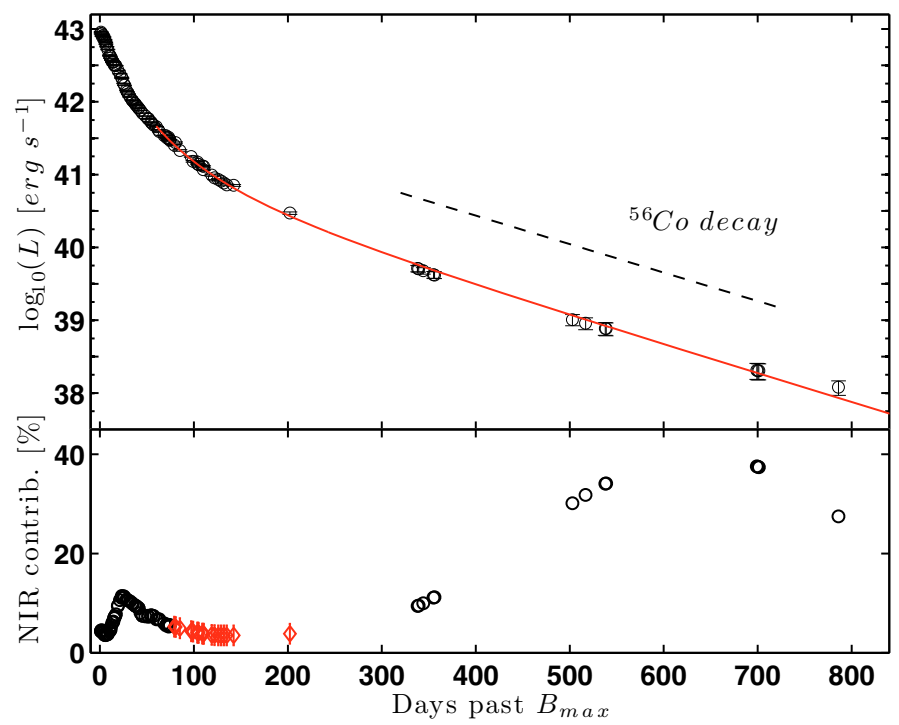

Fig. 8. Upper panel: the UVOIR light curve of SN 2003hv constructed as described in Sect. 5.4. The last point is based mostly on extrapolations, and should be regarded as rather uncertain. The red solid line is the best fit of a radioactive decay energy deposition function of ${ }^{56} \mathrm{Co}$ between +60 and 700 days. The estimated ${ }^{56} \mathrm{Ni}$ mass from the energy deposition function fit is $0.22 \pm 0.02 M_{\odot}$, which is less than what was obtained at maximum light $\left(0.40 \pm 0.07 M_{\odot}\right)$, indicating that at these late phases a substantial fraction of the flux is emitted outside the UVOIR bands. At 300-700 days past maximum the decline rate is linear and follows the expected decay time of ${ }^{56} \mathrm{Co}$ (dashed line) assuming full and instantaneous positron energy deposition. The displayed error bars do not include the error in the distance to NGC 1201. Lower panel: The evolution of the NIR contribution to the UVOIR light curve, computed as integrated flux from $J$ to $K$ over the integrated UVOIR flux. Since no NIR data were available at $+60-200$ days, we assumed a contribution (red diamonds in the graph) that maintains the smoothness of the curve and is compatible with the corresponding temporal evolution observed by S04.

applied for the flux lost blueward of $U$ or redward of $K$. The UVOIR light curve is displayed in Fig. 8.

The UVOIR light curve can be used to estimate the amount of ${ }^{56} \mathrm{Ni}$ synthesized during the explosion. Using the estimate of the peak brightness and application of Arnett's rule (Arnett 1982) suggests that $0.40-0.42 M_{\odot}$ of ${ }^{56} \mathrm{Ni}$ was synthesized in the explosion, depending on whether we choose to correct by an additional $5 \%$ or $10 \%$ for the flux not observed blueward of the $U$ band (Stritzinger et al. 2006a, and references therein). The associated error amounts to $0.07 M_{\odot}$, accounting for the errors in the measured flux and the uncertainty in the rise time. The error increases to $0.11 M_{\odot}$ if the uncertainty in the distance to NGC 1201 is included.

The ${ }^{56} \mathrm{Ni}$ mass can also be estimated by fitting an energy deposition function for the radioactive decay of ${ }^{56} \mathrm{Co}$ in the tail of the bolometric light curve. A simple model, also used by S04 and $\mathrm{SS} 07$, is $L=1.3 \times 10^{43} M_{\mathrm{Ni}} \mathrm{e}^{-t / 111.3}\left(1-0.966 \mathrm{e}^{-\tau}\right)$, where $L$ is the bolometric luminosity, $M_{\mathrm{Ni}}$ is the ${ }^{56} \mathrm{Ni}$ mass, $t$ is the time past maximum, and $\tau$ is the optical depth which is taken to evolve as $\left(t_{1} / t\right)^{2}$, where $t_{1}$ is the time where the optical depth to the gamma rays becomes unity (Sollerman et al. 1998). This model assumes complete and instantaneous energy deposition from the positrons. By fitting this simple equation to our UVOIR light curve in the range $+60-700$ days, we obtain $M_{\mathrm{Ni}}=0.22 \pm 0.02 M_{\odot}$ and $t_{1}=$ $32.7 \pm 1.8$ days. The quoted errors here are merely the formal errors from the least-square fits. Since no $J H K$ observations were available at +60-200 days, we made an assumption for the NIR contribution $^{9}$ at these phases (Fig. 8, lower panel). The adopted assumption ensures that the evolution of the NIR contribution is smooth and similar to that of the well-observed SN 2000cx (S04); that is, we have assumed that the NIR contribution continues its smooth decline until it reaches a minimum at around +130 days, after which there is an upturn that slowly leads to the observed high NIR contribution at late times. An associated uncertainty of $\pm 2 \%$ was assumed for these calculations. In addition, it was checked that other reasonable assumptions do not change the derived ${ }^{56} \mathrm{Ni}$ mass by more than $\pm 0.02 M_{\odot}$.

We point out that there is a substantial difference between the ${ }^{56} \mathrm{Ni}$ mass estimated from the energy emitted in the UVOIR bands around maximum light compared to that estimated at late phases. There could be a number of reasons for this "missing flux", including color evolution outside the UVOIR bands, positron escape, or an IRC.

Previous studies have shown that color evolution is very important at these stages within the UVOIR limits: S04 demonstrated that the contribution of the NIR bands to the UVOIR luminosity increased from about $3 \%$ to $28 \%$ between +150 and +500 days. A similar result was obtained by SS07. We find that this fraction for SN $2003 \mathrm{hv}$ evolves from about $9 \%$ to $30 \%$ between +330 and +500 days and increases to $\sim 37 \%$ by +700 days (Fig. 8, lower panel).

S04 and SS07 also estimated that the UVOIR light curve might probe only $\sim 60 \%$ of the true bolometric luminosity at these late phases. Our modeling suggests that at +350 days the UVOIR misses $27 \%$ of the total luminosity, while at +500 days this ratio increases to $44 \%$. It is most likely that the difference in derived nickel mass is due to a significant fraction of flux at late phases being emitted in the far-IR.

By integrating the flux contained within the nebular spectra in Fig. 7, we find that the flux emitted in the MIR region probed by Spitzer is a significant portion of the total flux probed at day +358: $L_{\mathrm{MIR}} / L_{\mathrm{tot}_{\mathrm{probed}}}=(34 \pm 17) \%$. The large error bar is due to the poor signal-to-noise ratio of the MIR spectrum, and there is an additional uncertainty in the Spitzer absolute-flux calibration. For comparison, our model, for which the onset of the IRC occurs later (i.e., 500-700 days), predicts only a value of $8 \%$ for this ratio. Note also that the probed MIR range is still blueward of most fine-structure lines in the far-IR, to which most of the flux would shift in the event of an IRC.

\subsection{Trapping of positrons}

There is an ongoing debate concerning the extent to which the positrons created in the radioactive decays are trapped in the ejecta. This discussion has implications for our understanding of the magnetic field configuration of the ejecta (Ruiz-Lapuente \& Spruit 1998). It is of interest for understanding the Galactic $511 \mathrm{keV}$ emission where SNe Ia have been suspected to contribute if the positrons escape (Milne et al. 1999; Prantzos 2008), and it is also important in order to properly model the late-time emission of SNe Ia. Our spectral synthesis model assumes that all positrons are trapped and that they deposit their energy in situ, but more elaborate positron transfer mechanisms could be envisioned (Milne et al. 1999).

The most straightforward observational test is to measure the decline rate of the late-time bolometric light curve, since positrons are the main energy contributors during late phases when the gamma rays escape the ejecta freely. In the simplest

\footnotetext{
9 By NIR contribution to UVOIR, we define here the ratio of the integrated flux from $J$ through $K$ to the integrated flux from $U$ through $K$.
} 
scenario, complete positron trapping will result in a late-phase bolometric light curve that follows the decay rate of radioactive ${ }^{56} \mathrm{Co}$ (0.98 mag per 100 days), whereas positron escape would produce a faster decay rate. In particular, one would expect positron escape to become increasingly important at later phases, and therefore a bolometric light curve that deviates progressively more from the radioactive input rate (e.g., Milne et al. 2001, their Fig. 1).

The late-time UVOIR decline rate of SN 2003hv between +300 and +700 days is $0.99 \pm 0.04$ mag per 100 days, exactly what is expected for complete and instantaneous positron trapping (Fig. 8). In addition, during these epochs, the UVOIR light curve does not show large deviations from a linear decay. Only observations past day +300 were included to minimize contamination from energy deposited by gamma rays, and the data point at +786 days was ignored because it is based mainly on extrapolations (except in the $H$-band).

This gives little room for energy being lost in the form of positrons, at least between +300 and 700 days. There can, in principle, be alternative explanations that give a shallower light curve while allowing for positrons to escape, such as freeze-out (Fransson \& Kozma 1993). However, this would require multiple effects to "conspire" to give an extended linear decay with a slope that perfectly mimics that of radioactive decay. We therefore believe that there is no evidence for substantial leakage; it is adequate to model $\mathrm{SNe}$ Ia at late times assuming that positrons do not escape.

Turning to the contribution of positrons to the Galactic $511 \mathrm{keV}$ line, Prantzos (2008) mentions that a constant escape fraction as small as 3\% would be enough to make them an important source. It is difficult to exclude such a small contribution. We merely note that as our light curves show little evidence for positron escape at late phases, it is hard to imagine that positron escape is important at earlier epochs when the density of the ejecta is considerably higher and the magnetic field strength is greater. Of course, SNe Ia do show some diversity at late times (e.g., the peculiar SN 2006gz; Maeda et al. 2009a), but our conclusions seem to hold for SN 2000cx (S04), SN 2001el (SS07), and SN 2003hv.

We already noted that within the electron capture scenario favored by Motohara et al. (2006) to explain the flat-topped line profiles, there is additional evidence for in situ positron trapping. If the positrons from the radioactive isotopes were able to travel inside the ejecta, they would also excite the central material and not give rise to a flat-topped profile.

\subsection{IRC}

Having discussed the positron trapping and the missing flux at late times in the UVOIR light curve, we now proceed to the question of whether an IRC could have occurred in the ejecta of SN 2003hv.

Figure 9 shows the late $U B V R I J H K$ light curves of SN 2003hv together with our detailed model light curves based on the W7 model (see also S04). The very sharp drop in the modeled light curves between +550 and 700 days is a consequence of the IRC.

From the modeling point of view, the IRC is expected to occur once the temperature of the ejecta drops below a critical threshold. In the case of SN 2003hv, the observed light curves appear to show little evidence of such a dramatic scenario. Not only do the optical bands seem to demonstrate an opposite trend, as discussed above, but the +786 day $H$-band detection places strong constraints on the drop of the NIR luminosity. While this has been hinted by single optical passband observations in the past, this is the first multi-wavelength study extending to such late phases.

The lack of a sudden and dramatic drop in the late-phase flux suggests that at least a portion of the ejecta is kept above the critical temperature limit which marks the onset of the IRC. One possible solution that we propose here is clumping of the ejecta. In this case, lower density regions cool more slowly and remain hot enough to avoid reaching the IRC temperature limit. The emission from these regions may dominate the optical and NIR range, thus causing the flux of the SN not to decrease as fast as predicted by a model with a more homogeneous density distribution. This is illustrated in Fig. 9 by the dashed curve, which represents a model with clumpy ejecta. In this simplistic model, clumping is achieved by artificially compressing and decompressing subsequent layers of the W7 ejecta. We stress that no effort has been made to "fit" the data or to simulate a realistic 3-D situation. The purpose of this experiment is to show that clumping can postpone the main observational signatures of an IRC. However, even in this model the high-density regions cool and indeed undergo the IRC. The lower density regions, on the other hand, stay hot enough for this not to occur and therefore continue to emit sufficient flux in the optical and NIR.

Support for the inhomogeneous nature of the cooling comes from the calculations by Kozma et al. (2005), based on the 3-D model by Röpke (2005): at +500 days, a large range of temperatures (and ionizations) is present, from a few hundred $\mathrm{K}$ to $\gtrsim 6000 \mathrm{~K}$ (their Fig. 7). Whether the IRC occurs in a specific region depends both on the density and composition. The dependence on composition comes mainly from the difference in ${ }^{56} \mathrm{Ni}$ content, which affects the local heating by the positrons.

One way to interpret our observations could thus be to propose that an IRC has taken place in the highest density regions of the ejecta. With the innermost regions having higher densities, this scenario can explain the lack of emission in these regions, resulting in the observed flat-topped line profiles. This idea can also explain the missing flux in the tail of the UVOIR light curve as estimated from the difference of the derived ${ }^{56} \mathrm{Ni}$ masses at maximum and at late phases. It is, however, required that the highest density regions must have already suffered this IRC before $~ 350-$ 700 days, since during these epochs the UVOIR light curve shows little deviation from the ${ }^{56}$ Co radioactive decay rate of 0.98 mag per 100 days. The less-dense parts still continue emitting in the optical and NIR wavelengths. This scenario is also supported by the excess flux measured in the MIR at +358 days.

Further pursuit of the idea of clumpiness is beyond the scope of this paper, but we suggest this to be an interesting topic of investigation for future 3-D modeling efforts. Here we restrict ourselves to pointing out that an IRC might have occurred in the innermost, highest density regions of SN 2003hv, and that this idea is compatible with the multi-wavelength observations of the ejecta.

\subsection{Geometry of the explosion}

As also pointed out by Motohara et al. (2006), the blueshifted lines observed in some of the emission features of the nebular spectrum may suggest an asymmetry in the distribution of ${ }^{56} \mathrm{Ni}$ that was formed during the explosion. This is also the conclusion of Maeda et al. (2009b), who model the nebular spectra of SN 2003hv with the aid of a simplified kinematical model and propose that we are viewing the explosion toward an offset high-density region. Hillebrandt et al. (2007) and Sim et al. (2007) showed that the viewing angle of off-centre explosions can have a significant effect on the light-curve properties 


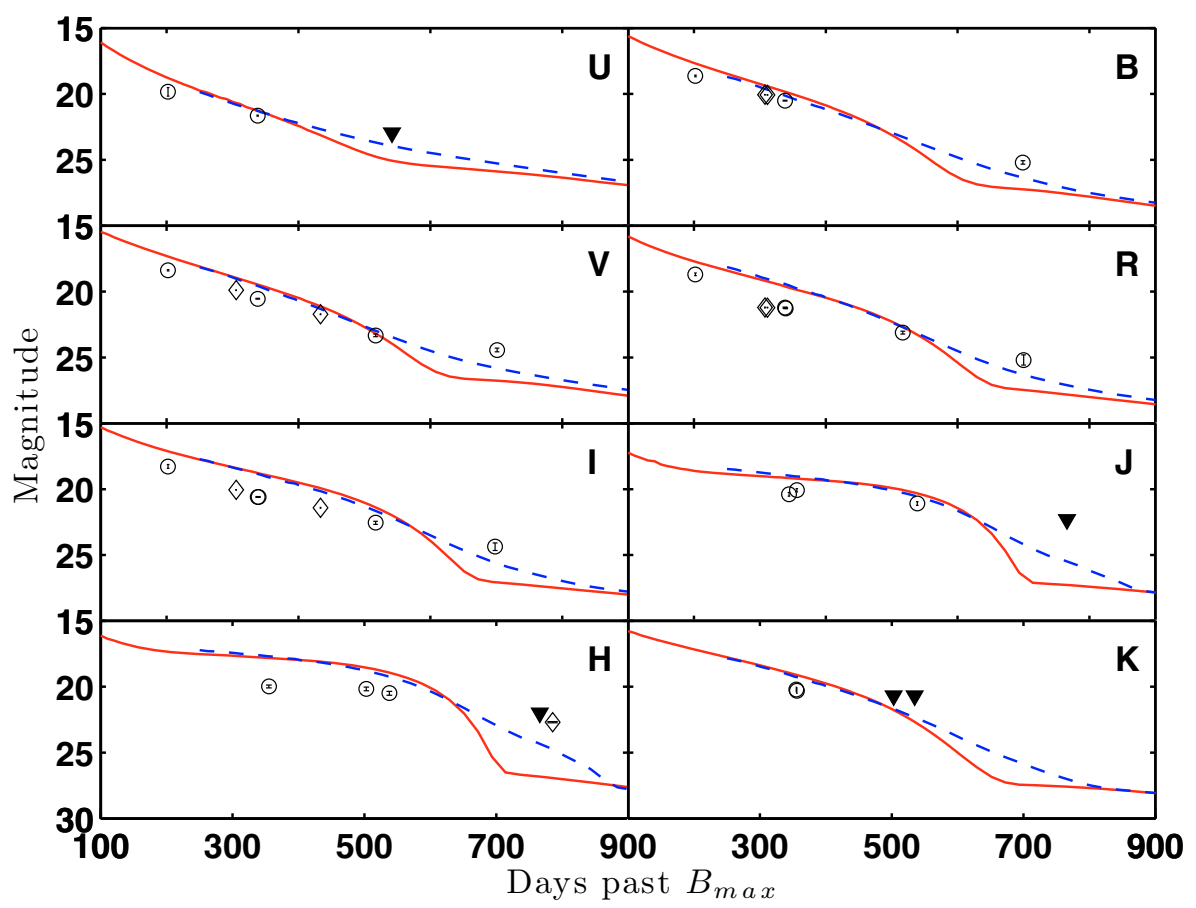

Fig. 9. Photometric data of SN 2003hv after 200 days past maximum compared with the model light curves of W7 and our spectral synthesis code (solid red line). Circles are groundbased data, diamonds are data from HST, and filled triangles denote upper limits. The sharp drops in the model light curves between +550 and +700 days are due to the predicted IRC. The observed data do not show such a dramatic evolution. The dashed blue line is a simplified "clumped" model illustrating how clumping can reduce or postpone the effects of the IRC: the high-density regions undergo an IRC first, but the low-density regions remain hot and continue emitting at the UVOIR wavelengths. of SNe Ia. An enhancement in brightness is expected in their models when the bulk of ${ }^{56} \mathrm{Ni}$ is moving toward the observer. However, this is not observed in SN 2003hv, which has a normal luminosity.

A different diagnostic of (another kind of) asymmetry comes from the analysis of spectropolarimetry of SN $2003 \mathrm{hv}$ at +6 days (unpublished data). While the lack of continuum polarization suggests that the photosphere was spherical at these times, nonnegligible line polarization $(0.19 \%)$ associated with Fe II and $\mathrm{Si}$ II features implies an asymmetric or clumpy line-forming region for these two species. Note that the polarization of the Si II feature at these times is at odds with the suggested evolutionary trend of Si II polarization in normal SNe Ia, for which zero polarization is expected at +6 days after maximum (e.g., SNe 2001el, 2002bo, see Wang et al. 2003, 2007). Despite the fact that these two observations probe completely different parts of the ejecta, and it is very difficult to link them in a common conclusion, they might hint that a simple spherically symmetric explosion cannot accurately describe SN 2003hv. This is not incompatible with our idea of clumping. A complete treatment and analysis of the spectropolarimetry will be given by Maund et al. (2009).

\section{Summary and conclusions}

We have presented observations of SN 2003hv that were obtained with a multitude of instruments. This study includes systematic multi-band observations from early to very late phases, and the latest-ever detection of a SN Ia in the NIR. It also features a comparison of our nebular spectrum synthesis model with the widest (in wavelength coverage) nebular spectrum of a SN Ia.

SN 2003hv is a SN Ia with the unusual value of $\Delta m_{15}(B)=$ $1.61 \pm 0.02 \mathrm{mag}$, and it exhibits photometric and spectroscopic properties that are consistent with its decline-rate parameter.

The main conclusions of the late-phase study are as follows:

- The individual light curves have decline rates similar to what has been observed for other SNe Ia in the past, confirming that there is an evolution of the flux from the optical to the NIR wavelengths.
- At +700 days, a deceleration in the fading of the SN emission is observed in the individual optical bands, particularly in the $V$-band. Such tendencies have been seen in other SNe Ia observed past 600 days.

- By comparing the radioactive energy input to the ejecta, as expressed from the ${ }^{56} \mathrm{Ni}$ masses derived from the UVOIR light curve at maximum light and in the tail, we find that the amount of energy probed within the UVOIR wavelengths is substantially less at late times than at maximum brightness.

- A possible explanation for this could be that positrons escape the ejecta, thus stealing energy away from it. The UVOIR light curve, however, follows very accurately the radioactive decay of ${ }^{56} \mathrm{Co}$ in the range $+300-700$ days, assuming complete and instantaneous positron trapping. This slope is difficult to reconcile with positron escape. Alternatively, the UVOIR light curve is not a good probe of the real bolometric light curve at late times, because the energy is still within the ejecta but a significant part of it is emitted at even longer wavelengths.

- A hypothesis proposed here to explain the SN 2003hv data is that an IRC has occurred in the densest (i.e., innermost) part of the ejecta. This idea can explain i) the missing flux in the tail of the UVOIR curve, ii) the flat-topped NIR profiles, and iii) the excess flux observed in the MIR spectrum. However, such an IRC must have taken place before 2300-350 days, since this is when the slope settles down, the flat-topped profiles start to emerge, and the Spitzer observation took place.

- The notion of an IRC occurring locally (and not simultaneously all over the SN ejecta) is consistent with models that feature a clumped (or inhomogeneous) distribution of the ejecta. The high-density regions undergo the IRC first, since it is these that experience the most efficient cooling.

- Deviations from spherical symmetry are suggested for SN 2003hv by the blueshifts of the iron-family element lines from the optical to the MIR and, independently, from early spectropolarimetric observations. 
Acknowledgements. The Dark Cosmology Centre is funded by the Danish National Research Foundation. M. S. acknowledges support from the National Science Foundation (NSF) under grant AST-0306969. J. S. is a Royal Swedish Academy of Sciences Research Fellow supported by a grant from the Knut and Alice Wallenberg Foundation. A. V. F.'s group at UC Berkeley is supported by NSF grant AST-0607485 (UC Berkeley), by the TABASGO Foundation, and by NASA/HST grants GO-10272, AR-10952, and AR-11248 from STScI. KAIT and its ongoing operation were made possible by donations from Sun Microsystems, Inc., the Hewlett-Packard Company, AutoScope Corporation, Lick Observatory, the NSF, the University of California, the Sylvia \& Jim Katzman Foundation, and the TABASGO Foundation. A. V. F. thanks the Aspen Center for Physics, where he participated in a workshop on WideFast-Deep Surveys while this paper was nearing completion. C. F. acknowledges support from the Swedish National Space Board and the Swedish Research Council. M. H. is grateful for support provided by NASA through Hubble Fellowship grant HST-HF-01139.01-A (awarded by the Space Telescope Science Institute [STScI], which is operated for NASA by the Association of Universities for Research in Astronomy, Inc., under contract NAS 5-26555), the Carnegie Postdoctoral Fellowship, FONDECYT through grant 1060808, the Millennium Center for Supernova Science through grant P06-045-F (funded by "Programa Bicentenario de Ciencia y Tecnología de CONICYT" and "Programa Iniciativa Científica Milenio de MIDEPLAN"), Centro de Astrofísica FONDAP 15010003, and Center of Excellence in Astrophysics and Associated Technologies (PFB 06). The SSO observations were obtained as part of the European Union's Human Potential Program "The Physics of Type Ia Supernovae" under contract HPRN-CT-2002-00303. This research has made use of the Two Micron All-Sky Survey (2MASS), and the NASA/IPAC Extragalactic Database (NED), which is operated by the Jet Propulsion Laboratory, California Institute of Technology, under contract with NASA. We acknowledge Bruno Leibundgut and Peter Lundqvist for early discussions on this project. We are extremely grateful to Chris Gerardy for providing us with the Spitzer data, to Kentaro Motohara for sending us the Subaru NIR spectrum, and to Dietrich Baade and collaborators for providing an advance look at their data from ESO program 71.D-0141(A). G. L. wishes to thank Ken Nomoto for useful discussions on SN 2003hv in Cefalù, Sicily, and Keiichi Maeda for reading the manuscript and providing us with comments. We are also grateful to Sergio Gonzalez, Nidia Morrell, and Miguel Roth for obtaining some observations of SN 2003hv. Finally, we thank the referee, M. Turatto, for providing useful comments.

\section{References}

Arnett, W. D. 1982, ApJ, 253, 785

Axelrod, T. S. 1980, Ph.D. Thesis, California Univ., Santa Cruz

Barker, E., Dahlen, T., Koekemoer, A., et al. 2007, NICMOS Instrument Handbook, Version 10.0 (Baltimore: STScI)

Benetti, S., Meikle, P., Stehle, M., et al. 2004, MNRAS, 348, 261

Benetti, S., Cappellaro, E., Mazzali, P. A., et al. 2005, ApJ, 623, 1011

Bessell, M. S. 1990, PASP, 102, 1181

Beutler, B., \& Li, W. 2003, IAU Circ., 8197, 1

Blondin, S., \& Tonry, J. L. 2007, ApJ, 666, 1024

Burns et al. 2009, in preparation

Cappellaro, E., Mazzali, P. A., Benetti, S., et al. 1997, A\&A, 328, 203

Cappellaro, E., Patat, F., Mazzali, P. A., et al. 2001, ApJ, 549, L215

Colgate, S. A., Petschek, A. G., \& Kriese, J. T. 1980, ApJ, 237, L81

Crotts, A. P. S. \& Yourdon, D. 2008, ApJ, 689, 1186

Devillard, N. 1999, in Astronomical Data Analysis Software and Systems VIII, ed. D. M. Mehringer, R. L. Plante, \& D. A. Roberts, ASP Conf. Ser., 172, 333 Dressler, A., Phillips, M., Morrell, N., \& Hamuy, M. 2003, IAU Circ., 8198, 2 Filippenko, A. V., Richmond, M. W., Branch, D., et al. 1992, AJ, 104, 1543 Filippenko, A. V., Li, W. D., Treffers, R. R., \& Modjaz, M. 2001, in IAU Colloq. 183: Small Telescope Astronomy on Global Scales, ed. B. Paczynski, W.-P. Chen, \& C. Lemme, ASP Conf. Ser., 246, 121

Fransson, C., \& Kozma, C. 1993, ApJ, 408, L25

Freedman, W. L., Madore, B. F., Gibson, B. K., et al. 2001, ApJ, 553, 47

Garnavich, P. M., Bonanos, A. Z., Krisciunas, K., et al. 2004, ApJ, 613, 1120

Gerardy, C. L., Meikle, W. P. S., Kotak, R., et al. 2007, ApJ, 661, 995

Hamuy, M., Trager, S. C., Pinto, P. A., et al. 2000, AJ, 120, 1479

Hamuy, M., Folatelli, G., Morrell, N. I., et al. 2006, PASP, 118, 2

Hamuy, M., Deng, J., Mazzali, P. A., et al. 2009, ApJ, in press

[arXiv:0908.1783]

Hillebrandt, W. \& Niemeyer, J. C. 2000, ARA\&A, 38, 191
Hillebrandt, W., Sim, S. A., \& Röpke, F. K. 2007, A\&A, 465, L17 Höflich, P., Gerardy, C. L., Nomoto, K., et al. 2004, ApJ, 617, 1258 Hsiao, E. Y., Conley, A., Howell, D. A., et al. 2007, ApJ, 663, 1187 Jensen, J. B., Tonry, J. L., Barris, B. J., et al. 2003, ApJ, 583, 712 Jha, S., Riess, A. G., \& Kirshner, R. P. 2007, ApJ, 659, 122 Kozma, C. \& Fransson, C. 1998a, ApJ, 496, 946 Kozma, C. \& Fransson, C. 1998b, ApJ, 497, 431 Kozma, C., Fransson, C., Hillebrandt, W., et al. 2005, A\&A, 437, 983 Krisciunas, K., Suntzeff, N. B., Candia, P., et al. 2003, AJ, 125, 166 Krisciunas, K., Phillips, M. M., Suntzeff, N. B., et al. 2004, AJ, 127, 1664 Krisciunas, K., Marion, H., Suntzeff, N. B., et al. 2009, [arXiv: 0908. 1918] Lair, J. C., Leising, M. D., Milne, P. A., \& Williams, G. G. 2006, AJ, 132, 2024 Landolt, A. U. 1992, AJ, 104, 340

Leloudas, G., Stritzinger, M., Sollerman, J., Hamuy, M., \& Suntzeff, N. 2009, in American Institute of Physics Conference Series, ed. G. Giobbi, A. Tornambe, G. Raimondo, M. Limongi, L. A. Antonelli, N. Menci, \& E. Brocato, ASP Conf. Ser., 1111, 456

Maeda, K., Kawabata, K., Li, W., et al. 2009a, ApJ, 690, 1745

Maeda, K., Taubenberger, S., Sollerman, J., et al. 2009b, ApJ, submitted

Maund, J. R., et al. 2009, in preparation

Mazzali, P. A., Cappellaro, E., Danziger, I. J., Turatto, M., \& Benetti, S. 1998, ApJ, 499, L49+

Mazzali, P. A., Röpke, F. K., Benetti, S., \& Hillebrandt, W. 2007, Sci, 315, 825 McLaughlin, H., \& Wiklind, T. 2007, NICMOS Data Handbook, Version 7.0 (Baltimore: STScI)

Milne, P. A., The, L.-S., \& Leising, M. D. 1999, ApJS, 124, 503

Milne, P. A., The, L.-S., \& Leising, M. D. 2001, ApJ, 559, 1019

Milne, P. A. \& Williams, G. G. 2005, in Cosmic Explosions, On the 10th Anniversary of SN1993J, ed. J.-M. Marcaide, \& K. W. Weiler, IAU Colloq., 192,183

Motohara, K., Maeda, K., Gerardy, C. L., et al. 2006, ApJ, 652, L101

Nomoto, K., Thielemann, F.-K., \& Yokoi, K. 1984, ApJ, 286, 644

Nugent, P., Phillips, M., Baron, E., Branch, D., \& Hauschildt, P. 1995, ApJ, 455, L147+

Nugent, P., Kim, A., \& Perlmutter, S. 2002, PASP, 114, 803

Pakmor, R., Röpke, F. K., Weiss, A., \& Hillebrandt, W. 2008, A\&A, 489, 943

Pastorello, A., Taubenberger, S., Elias-Rosa, N., et al. 2007, MNRAS, 376, 1301 Patat, F. 2005, MNRAS, 357, 1161

Pavlovsky, C., Koekemoer, A., \& Mack, J. 2006, ACS Data Handbook, Version 5.0 (Baltimore: STScI)

Persson, S. E., Murphy, D. C., Krzeminski, W., Roth, M., \& Rieke, M. J. 1998, AJ, 116, 2475

Phillips, M. M. 1993, ApJ, 413, L105

Phillips, M. M., Lira, P., Suntzeff, N. B., et al. 1999, AJ, 118, 1766

Pignata, G., Benetti, S., Mazzali, P. A., et al. 2008, MNRAS, 388, 971

Prantzos, N. 2008, NAR, 52, 457

Prieto, J. L., Rest, A., \& Suntzeff, N. B. 2006, ApJ, 647, 501

Röpke, F. K. 2005, A\&A, 432, 969

Röpke, F. K., Hillebrandt, W., Schmidt, W., et al. 2007, ApJ, 668, 1132

Ruiz-Lapuente, P. \& Spruit, H. C. 1998, ApJ, 500, 360

Schlegel, D. J., Finkbeiner, D. P., \& Davis, M. 1998, ApJ, 500, 525

Schmidt, B. P., Kirshner, R. P., Leibundgut, B., et al. 1994, ApJ, 434, L19

Sim, S. A., Sauer, D. N., Röpke, F. K., \& Hillebrandt, W. 2007, MNRAS, 378, 2 Sirianni, M., Jee, M. J., Benítez, N., et al. 2005, PASP, 117, 1049

Sollerman, J., Leibundgut, B., \& Spyromilio, J. 1998, A\&A, 337, 207

Sollerman, J., Lindahl, J., Kozma, C., et al. 2004, A\&A, 428, 555

Spyromilio, J., \& Graham, J. R. 1992, MNRAS, 255, 671

Stanishev, V., Goobar, A., Benetti, S., et al. 2007, A\&A, 469, 645

Stritzinger, M. \& Sollerman, J. 2007, A\&A, 470, L1

Stritzinger, M., Hamuy, M., Suntzeff, N. B., et al. 2002, AJ, 124, 2100

Stritzinger, M., Suntzeff, N. B., Hamuy, M., et al. 2005, PASP, 117, 810

Stritzinger, M., Leibundgut, B., Walch, S., \& Contardo, G. 2006a, A\&A, 450, 241

Stritzinger, M., Mazzali, P. A., Sollerman, J., \& Benetti, S. 2006b, A\&A, 460, 793

Suntzeff, N. B. 1996, in Supernovae and Supernova Remnants, IAU Colloq., 145, 41

Tonry, J. L., Dressler, A., Blakeslee, J. P., et al. 2001, ApJ, 546, 681

Wang, L., Baade, D., Höflich, P., et al. 2003, ApJ, 591, 1110

Wang, L., Baade, D., \& Patat, F. 2007, Sci, 315, 212

Wang, X., Li, W., Filippenko, A. V., et al. 2009, ApJ, 697, 380

Wang, X., Li, W., Filippenko, A. V., et al. 2008, ApJ, 677, 1060 
G. Leloudas et al.: The normal Type Ia SN 2003hv out to very late phases, Online Material p 1

Table 3. S-corrections (mag) computed for phases up to +70 days ${ }^{a}$.

\begin{tabular}{crrrr}
\hline \hline MJD & $B$ & $V$ & $R$ & $I$ \\
\hline 52892.40 & 0.010 & 0.022 & 0.014 & -0.034 \\
52892.47 & -0.003 & 0.002 & 0.036 & -0.017 \\
52893.39 & 0.010 & 0.021 & 0.015 & -0.033 \\
52893.41 & -0.004 & 0.003 & 0.036 & -0.018 \\
52894.37 & 0.010 & 0.020 & 0.016 & -0.032 \\
52895.37 & 0.011 & 0.017 & 0.017 & -0.031 \\
52896.38 & 0.013 & 0.013 & 0.018 & -0.035 \\
52896.52 & -0.005 & 0.007 & 0.037 & -0.018 \\
52897.37 & 0.015 & 0.009 & 0.019 & -0.038 \\
52897.51 & -0.005 & 0.008 & 0.036 & -0.018 \\
52898.52 & -0.006 & 0.009 & 0.034 & -0.018 \\
52899.52 & -0.006 & 0.010 & 0.032 & -0.018 \\
52901.40 & 0.000 & 0.013 & 0.014 & 0.029 \\
52901.44 & -0.010 & 0.010 & 0.027 & -0.019 \\
52902.30 & -0.004 & 0.014 & 0.014 & 0.036 \\
52902.48 & -0.016 & 0.010 & 0.025 & -0.021 \\
52903.46 & -0.020 & 0.009 & 0.024 & -0.022 \\
52904.49 & -0.022 & 0.009 & 0.021 & -0.023 \\
52905.26 & 0.034 & -0.009 & 0.007 & -0.054 \\
52905.30 & -0.012 & 0.015 & 0.010 & 0.057 \\
52906.40 & -0.011 & 0.016 & 0.009 & 0.066 \\
52906.48 & -0.018 & 0.008 & 0.018 & -0.025 \\
52907.30 & -0.011 & 0.016 & 0.008 & 0.072 \\
52908.30 & -0.012 & 0.016 & 0.008 & 0.078 \\
52910.47 & -0.020 & 0.006 & 0.012 & -0.026 \\
52912.47 & -0.023 & 0.007 & 0.013 & -0.024 \\
52914.40 & -0.015 & 0.015 & 0.004 & 0.084 \\
52914.46 & -0.028 & 0.009 & 0.015 & -0.020 \\
52916.46 & -0.024 & 0.011 & 0.017 & -0.018 \\
52919.42 & -0.014 & 0.015 & 0.018 & -0.014 \\
52921.40 & -0.012 & 0.017 & 0.019 & -0.010 \\
52925.43 & -0.011 & 0.019 & 0.020 & -0.007 \\
52928.42 & -0.015 & 0.019 & 0.024 & -0.007 \\
52929.40 & -0.004 & 0.022 & 0.005 & 0.096 \\
52930.42 & -0.020 & 0.018 & 0.028 & -0.007 \\
52932.37 & -0.021 & 0.018 & 0.030 & -0.006 \\
52934.41 & -0.019 & 0.018 & 0.031 & -0.005 \\
52936.40 & -0.015 & 0.018 & 0.030 & -0.005 \\
52939.36 & -0.011 & 0.018 & 0.029 & -0.004 \\
52942.36 & -0.007 & 0.017 & 0.026 & -0.002 \\
52942.66 & -0.000 & -0.007 & -0.004 & 0.046 \\
52945.38 & -0.005 & 0.017 & 0.024 & -0.000 \\
52948.32 & -0.004 & 0.016 & 0.023 & -0.002 \\
52951.30 & 0.004 & 0.016 & 0.004 & 0.084 \\
52954.36 & -0.003 & 0.016 & 0.022 & -0.004 \\
52959.33 & 0.004 & 0.016 & 0.019 & -0.004 \\
52961.28 & 0.022 & 0.003 & 0.004 & 0.010 \\
\hline & & & & \\
\hline & &
\end{tabular}

${ }^{a}$ We have used these corrections everywhere in this paper by adding them to the corresponding values of Table 4 . 
Table 4. Optical photometry of SN $2003 \mathrm{hv}^{a}$.

\begin{tabular}{|c|c|c|c|c|c|c|c|c|}
\hline $\begin{array}{l}\text { Date } \\
\text { (UT) }\end{array}$ & $\begin{array}{c}\text { MJD } \\
\text { (days) }\end{array}$ & $\begin{array}{l}\begin{array}{l}\text { Phase } \\
\text { (days) }\end{array}\end{array}$ & 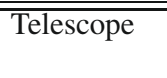 & $\begin{array}{c}U \\
(\mathrm{mag})\end{array}$ & $\begin{array}{c}B \\
(\mathrm{mag})\end{array}$ & $\begin{array}{c} \\
(\mathrm{mag})\end{array}$ & $\begin{array}{c}R \\
(\mathrm{mag})\end{array}$ & $\begin{array}{c}I \\
(\mathrm{mag})\end{array}$ \\
\hline 20030910 & 52892.40 & 1.2 & CTIO $0.9 \mathrm{~m}$ & $11.940(0.152)$ & $12.452(0.019)$ & $12.483(0.015)$ & $12.459(0.015)$ & $12.759(0.015)$ \\
\hline 20030910 & 52892.47 & 1.3 & KAIT & & $12.523(0.015)$ & $12.565(0.015)$ & $12.489(0.015)$ & $12.827(0.026)$ \\
\hline 20030911 & 52893.39 & 2.2 & CTIO $0.9 \mathrm{~m}$ & $12.125(0.102)$ & $12.505(0.020)$ & $12.510(0.015)$ & $12.477(0.015)$ & $12.779(0.015)$ \\
\hline 20030911 & 52893.41 & 2.2 & KAIT & & $12.619(0.015)$ & $12.616(0.015)$ & $12.509(0.015)$ & $12.870(0.019)$ \\
\hline 20030912 & 52894.37 & 3.2 & CTIO $0.9 \mathrm{~m}$ & $12.176(0.058)$ & $12.534(0.018)$ & $12.546(0.015)$ & $12.514(0.015)$ & $12.844(0.015)$ \\
\hline 20030913 & 52895.37 & 4.2 & CTIO $0.9 \mathrm{~m}$ & $12.131(0.063)$ & $12.631(0.015)$ & $12.573(0.015)$ & $12.586(0.015)$ & $12.949(0.015)$ \\
\hline 20030914 & 52896.38 & 5.2 & CTIO $0.9 \mathrm{~m}$ & $12.371(0.055)$ & $12.702(0.016)$ & $12.594(0.015)$ & $12.626(0.015)$ & $12.971(0.015)$ \\
\hline 20030914 & 52896.52 & 5.3 & KAIT & S & $12.750(0.015)$ & $12.676(0.015)$ & $12.693(0.015)$ & $13.040(0.020)$ \\
\hline 20030915 & 52897.37 & 6.2 & CTIO $0.9 \mathrm{~m}$ & $12.422(0.052)$ & $12.805(0.015)$ & $12.681(0.015)$ & $12.742(0.015)$ & $13.079(0.015)$ \\
\hline 20030915 & 52897.51 & 6.3 & KAIT & & $12.887(0.015)$ & $12.748(0.015)$ & $12.814(0.015)$ & $13.180(0.020)$ \\
\hline 20030916 & 52898.52 & 7.3 & KAIT & te & $12.985(0.015)$ & $12.804(0.015)$ & $12.911(0.015)$ & $13.263(0.023)$ \\
\hline 20030917 & 52899.52 & 8.3 & KAIT & & $13.094(0.015)$ & $12.858(0.015)$ & $13.010(0.015)$ & $13.288(0.024)$ \\
\hline 20030919 & 52901.40 & 10.2 & LCO Swope & $13.113(0.089)$ & $13.319(0.021)$ & $13.004(0.018)$ & & \\
\hline 20030919 & 52901.44 & 10.2 & KAIT & & $13.406(0.015)$ & $13.044(0.015)$ & $13.120(0.015)$ & $13.330(0.019)$ \\
\hline 20030920 & 52902.30 & 11.1 & LCO Swope & $13.358(0.018)$ & $13.410(0.015)$ & $13.010(0.015)$ & $13.083(0.015)$ & $13.201(0.015)$ \\
\hline 20030920 & 52902.48 & 11.3 & KAIT & & $13.520(0.015)$ & $13.055(0.015)$ & $13.175(0.015)$ & $13.327(0.017)$ \\
\hline 20030921 & 52903.46 & 12.3 & KAIT & & $13.664(0.015)$ & $13.134(0.015)$ & $13.193(0.015)$ & $13.317(0.019)$ \\
\hline 20030922 & 52904.49 & 13.3 & KAIT & & $13.789(0.015)$ & $13.247(0.015)$ & $13.233(0.015)$ & $13.277(0.019)$ \\
\hline 20030923 & 52905.26 & 14.1 & CTIO $0.9 \mathrm{~m}$ & $13.788(0.039)$ & $13.825(0.015)$ & $13.245(0.015)$ & $13.178(0.015)$ & $13.207(0.015)$ \\
\hline 20030923 & 52905.30 & 14.1 & LCO Swope & $13.838(0.040)$ & $13.851(0.015)$ & $13.215(0.015)$ & $13.118(0.015)$ & $13.128(0.015)$ \\
\hline 20030924 & 52906.40 & 15.2 & LCO Swope & $14.034(0.035)$ & $14.007(0.015)$ & $13.328(0.015)$ & $13.163(0.015)$ & $13.180(0.015)$ \\
\hline 20030924 & 52906.48 & 15.3 & KAIT & & $14.106(0.015)$ & $13.333(0.015)$ & $13.225(0.015)$ & $13.218(0.017)$ \\
\hline 20030925 & 52907.30 & 16.1 & LCO Swope & $62(0.0$ & $14.157(0.016)$ & $13.352(0.023)$ & $13.180(0.015)$ & $13.043(0.029)$ \\
\hline 20030926 & 52908.30 & 17.1 & LCO Swope & $14.249(0.038)$ & $14.298(0$ & $13.320(0.021)$ & $13.163(0.015)$ & $13.040(0.029)$ \\
\hline 20030928 & 52910.47 & 19.3 & KAIT & $\ldots$ & $14.621(0$. & $13.610(0$ & $13.360(C$ & $13.137(0.021)$ \\
\hline 20030930 & 52912.47 & 21.3 & KAIT & $\ldots$ & $14.808(0$ & $13.798(0.015)$ & $13.478(0.015)$ & $13.189(0.019)$ \\
\hline 20031002 & 52914.40 & 23.2 & LCO Swope & $15.020(0.102)$ & $14.992(0.022)$ & $13.925(0.015)$ & $13.561(0.020)$ & $13.212(0.015)$ \\
\hline 20031002 & 52914.46 & 23.3 & KAIT & $\cdots$ & $15.022(0.015)$ & $13.988(0.015)$ & $13.644(0.015)$ & $13.308(0.022)$ \\
\hline 20031004 & 52916.46 & 25.3 & KAIT & . & $15.158(0.015)$ & $14.141(0.015)$ & $13.844(0.015)$ & $13.512(0.022)$ \\
\hline 20031007 & 52919.42 & 28.2 & KAIT &. & $15.340(0.015)$ & $14.393(0.015)$ & $14.071(0.015)$ & $13.734(0.024)$ \\
\hline 20031009 & 52921.40 & 30.2 & KAIT & • & $15.448(0.015)$ & $14.480(0.015)$ & $14.189(0.015)$ & $13.913(0.025)$ \\
\hline 20031013 & 52925.43 & 34.2 & KAIT & & $15.640(0.021)$ & $14.658(0.022)$ & $14.392(0.020)$ & $14.143(0.030)$ \\
\hline 20031016 & 52928.42 & 37.2 & KAIT & $\cdots$ & $15.646(0.015)$ & $14.746(0.015)$ & $14.518(0.015)$ & $14.315(0.032)$ \\
\hline 20031017 & 52929.40 & 38.2 & LCO Swope & $15.511(0.045)$ & $15.624(0.015)$ & $14.781(0.015)$ & $14.523(0.015)$ & $14.325(0.015)$ \\
\hline 20031018 & 52930.42 & 39.2 & KAIT & $\cdots$ & $15.716(0.015)$ & $14.806(0.015)$ & $14.593(0.015)$ & $14.428(0.025)$ \\
\hline 20031020 & 52932.37 & 41.2 & KAIT & $\ldots$ & $15.735(0.015)$ & $14.871(0.015)$ & $14.671(0.015)$ & $14.566(0.022)$ \\
\hline 20031022 & 52934.41 & 43.2 & KAIT & $\ldots$ & $15.806(0.015)$ & $14.916(0.015)$ & $14.752(0.015)$ & $14.629(0.018)$ \\
\hline 20031024 & 52936.40 & 45.2 & KAIT & $\ldots$ & $15.843(0.015)$ & $15.018(0$ & $14.840(0.015)$ & $14.735(0.025)$ \\
\hline 20031027 & 52939.36 & 48.2 & KAIT &. & 15.929 & 15.094( & $14.926(0$ & $14.879(C$ \\
\hline 20031030 & 52942.36 & 51.2 & KAIT & . & $15.965(0$ & 15.199(C & $15.049(0$ & $15.043(0.031)$ \\
\hline 20031030 & 52942.66 & 51.5 & $\mathrm{SSO} 2.3 \mathrm{~m}$ & $15.792(0.039)$ & $15.952(0$. & $15.234(0.015)$ & $15.099(0$. & $14.877(0.017)$ \\
\hline 20031102 & 52945.38 & 54.2 & KAIT & $\ldots$ & $16.053(0.015)$ & $15.269(0.015)$ & $15.144(0.015)$ & $15.204(0.024)$ \\
\hline 20031105 & 52948.32 & 57.1 & KAIT & & $16.142(0.059)$ & $15.385(0.015)$ & $15.241(0.015)$ & $15.323(0.035)$ \\
\hline 20031108 & 52951.30 & 60.1 & LCO Swope & $16.110(0.035)$ & $16.086(0.015)$ & $15.408(0.015)$ & $15.310(0.015)$ & $15.379(0.015)$ \\
\hline 20031111 & 52954.36 & 63.2 & KAIT & 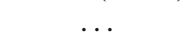 & $16.168(0.026)$ & $15.552(0.017)$ & $15.593(0.026)$ & \\
\hline 20031116 & 52959.33 & 68.1 & KAIT & $\ldots$ & $16.246(0.017)$ & $15.665(0.015)$ & $15.651(0.015)$ & $15.864(0.035)$ \\
\hline 20031118 & 52961.28 & 70.1 & CTIO $0.9 \mathrm{~m}$ & $\ldots$ & $16.260(0.015)$ & $15.667(0.015)$ & $15.644(0.015)$ & $15.855(0.015)$ \\
\hline 20031119 & 52962.34 & 71.1 & KAIT & $\ldots$ & $16.323(0.015)$ & $15.776(0.015)$ & $15.744(0.015)$ & $15.928(0.032)$ \\
\hline 20031120 & 52964.24 & 73.0 & VLT Antu & $\ldots$ & $16.277(0.042)$ & $15.793(0.015)$ & $15.582(0.024)$ & $16.015(0.015)$ \\
\hline 20031121 & 52964.32 & 73.1 & CTIO $0.9 \mathrm{~m}$ & $\ldots$ & $16.298(0.015)$ & $15.815(0.015)$ & $15.764(0.015)$ & $15.991(0.015)$ \\
\hline 20031122 & 52965.29 & 74.1 & CTIO $0.9 \mathrm{~m}$ & $\cdot$ & $16.347(0.015)$ & $15.819(0.015)$ & $15.797(0.015)$ & $16.036(0.015)$ \\
\hline 20031122 & 52965.31 & 74.1 & KAIT & & $16.338(0.015)$ & $15.855(0.018)$ & $15.846(0.015)$ & \\
\hline 20031127 & 52970.30 & 79.1 & KAIT & & $16.465(0.015)$ & $15.982(0.016)$ & $16.047(0.015)$ & 16.285 \\
\hline 20031128 & 52971.67 & 80.5 & $\mathrm{SSO} 2.3 \mathrm{~m}$ & $16.812(0.029)$ & $16.372(0.015)$ & $15.935(0.015)$ & $15.921(0.017)$ & $15.981(0.024)$ \\
\hline 20031203 & 52976.28 & 85.1 & KAIT & $\cdots$ & $16.576(0.033)$ & $16.186(0.039)$ & $16.244(0.030)$ & $16.519(0.065)$ \\
\hline 20031215 & 52988.30 & 97.1 & LCO Swope & $17.116(0.041)$ & $16.694(0.015)$ & $16.424(0.015)$ & $16.512(0.015)$ & $16.654(0.015)$ \\
\hline 20031217 & 52990.26 & 99.1 & KAIT & $\ldots$ & $16.836(0.024)$ & $16.615(0.035)$ & $16.706(0.048)$ & $16.854(0.087)$ \\
\hline 20031222 & 52995.10 & 103.9 & LCO Swope & $17.349(0.036)$ & & $16.569(0.015)$ & $16.736(0.015)$ & $16.840(0.016)$ \\
\hline 20031222 & 52995.24 & 104.0 & KAIT & 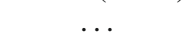 & $16.869(0.019)$ & $16.686(0.040)$ & $16.771(0.054)$ & $17.052(0.091)$ \\
\hline 20031223 & 52996.10 & 104.9 & LCO Swope & & $16.890(0.015)$ & & & \\
\hline 20031227 & 53000.10 & 108.9 & LCO Swope & $17.549(0.032)$ & $16.918(0.015)$ & $16.718(0.015)$ & $16.889(0.021)$ & $17.012(0.017)$ \\
\hline 20031228 & 53001.21 & 110.0 & KAIT & & $17.010(0.023)$ & $16.817(0.033)$ & $17.085(0.027)$ & $17.414(0.085)$ \\
\hline 20031228 & 53001.57 & 110.4 & $\mathrm{SSO} 2.3 \mathrm{~m}$ & $17.588(0.015)$ & $16.859(0.018)$ & $16.786(0.015)$ & $16.936(0.015)$ & $17.119(0.016)$ \\
\hline 20040106 & 53010.20 & 119.0 & KAIT & $\ldots$ & $17.120(0.052)$ & $17.107(0.047)$ & $17.336(0.048)$ & $17.316(0.100)$ \\
\hline 20040109 & 53013.19 & 122.0 & KAIT & $\ldots$ & $17.254(0.128)$ & $17.208(0.090)$ & $17.329(0.108)$ & \\
\hline 20040113 & 53017.18 & 126.0 & KAIT & $\ldots$ & $17.241(0.019)$ & $17.233(0.024)$ & $17.476(0.037)$ & $17.576(0.081)$ \\
\hline
\end{tabular}


G. Leloudas et al.: The normal Type Ia SN 2003hv out to very late phases, Online Material p 3

Table 4. (continued).

\begin{tabular}{ccclccccc}
\hline \hline $\begin{array}{c}\text { Date } \\
(\text { UT })\end{array}$ & $\begin{array}{c}\text { MJD } \\
(\text { days })\end{array}$ & $\begin{array}{c}\text { Phase } \\
(\text { days })\end{array}$ & Telescope & $\begin{array}{c}U \\
(\mathrm{mag})\end{array}$ & $\begin{array}{c}B \\
(\mathrm{mag})\end{array}$ & $\begin{array}{c}V \\
(\mathrm{mag})\end{array}$ & $\begin{array}{c}R \\
(\mathrm{mag})\end{array}$ & $\begin{array}{c}I \\
(\mathrm{mag})\end{array}$ \\
\hline 20040116 & 53020.16 & 129.0 & KAIT & $\ldots$ & $17.299(0.026)$ & $17.242(0.026)$ & $17.567(0.020)$ & $17.694(0.111)$ \\
20040119 & 53023.13 & 131.9 & KAIT & $\ldots$ & $17.349(0.028)$ & $17.347(0.026)$ & $17.625(0.041)$ & $17.706(0.072)$ \\
20040122 & 53026.15 & 135.0 & KAIT & $\ldots$ & $17.456(0.025)$ & $17.379(0.033)$ & $17.638(0.037)$ & $17.769(0.092)$ \\
20040129 & 53033.49 & 142.3 & SSO 2.3 m & $18.440(0.057)$ & $17.518(0.043)$ & $17.373(0.024)$ & $17.581(0.032)$ & $17.686(0.101)$ \\
20040329 & 53093.38 & 202.2 & SSO 2.3 m & $19.843(0.330)$ & $18.634(0.055)$ & $18.383(0.050)$ & $18.691(0.100)$ & $18.276(0.147)$ \\
20040710 & 53196.55 & 305.4 & HST ACS & $\ldots$ & $\ldots$ & $19.896(0.021)$ & $\ldots$ & $20.052(0.017)$ \\
20040712 & 53198.82 & 307.6 & HST ACS & $\ldots$ & $20.058(0.017)$ & $\ldots$ & $21.210(0.029)$ & $\ldots$ \\
20040716 & 53202.75 & 311.6 & HST ACS & $\ldots$ & $20.079(0.016)$ & $\ldots$ & $21.219(0.024)$ & $\ldots$ \\
20040812 & 53229.30 & 338.1 & VLT Kueyen & $21.648(0.039)$ & $20.508(0.028)$ & $20.543(0.033)$ & $21.224(0.042)$ & $20.587(0.032)$ \\
20040813 & 53230.39 & 339.2 & VLT Kueyen & $\ldots$ & $\ldots$ & $\ldots$ & $21.263(0.034)$ & $20.592(0.032)$ \\
20041115 & 53324.71 & 433.5 & HST ACS & $\ldots$ & $\ldots$ & $21.712(0.029)$ & $\ldots$ & $21.417(0.031)$ \\
20050207 & 53408.10 & 516.9 & VLT Antu & $\ldots$ & $\ldots$ & $23.326(0.100)$ & $23.111(0.102)$ & $22.554(0.122)$ \\
20050304 & 53433.02 & 541.8 & VLT Antu & $>22.95$ & $\ldots$ & $\ldots$ & $\ldots$ & $\ldots$ \\
20050808 & 53590.34 & 699.1 & VLT Kueyen & $\ldots$ & $25.198(0.137)$ & $\ldots$ & $\ldots$ & $\ldots$ \\
20050809 & 53591.36 & 700.2 & VLT Kueyen & $\ldots$ & $\ldots$ & $\ldots$ & $25.192(0.388)$ & $\ldots$ \\
20050810 & 53592.39 & 701.2 & VLT Kueyen & $\ldots$ & $\ldots$ & $24.423(0.138)$ & $\ldots$ & $\ldots$ \\
\hline
\end{tabular}

${ }^{a}$ The quoted values do not include S-corrections. Numbers in parentheses are uncertainties. All formal errors below 0.015 have been rounded up to this value.

${ }^{b}$ Vega magnitudes in the $H S T$ filter system $(F 435 W, F 555 W, F 625 W$, and $F 814 W$ ).

${ }^{c}$ In the last VLT epoch, two $R$ images and two $I$ images obtained at epochs differing by 5 and 1 days (respectively) have been combined to increase the signal-to-noise ratio. 
G. Leloudas et al.: The normal Type Ia SN 2003hv out to very late phases, Online Material p 4

\section{Appendix A: Observation Logs}

Table A.1. VLT late-time optical observations of SN 2003hv.

\begin{tabular}{cccccccc}
\hline \hline $\begin{array}{c}\text { Date } \\
(\mathrm{UT})\end{array}$ & $\begin{array}{c}\text { MJD } \\
\text { (days) }\end{array}$ & $\begin{array}{c}\text { Phase } \\
\text { (days) }\end{array}$ & $\begin{array}{c}\text { Filter } \\
\text { Exposure } \\
(\mathrm{s})\end{array}$ & Airmass & $\begin{array}{c}\text { Seeing } \\
(\operatorname{arcsec})\end{array}$ & Instrument \\
\hline 20040812 & 53229.33 & 338.1 & $U$ & $3 \times 1000$ & 1.17 & 1.02 & FORS1 \\
20040812 & 53229.37 & 338.2 & $B$ & $3 \times 420$ & 1.06 & 0.85 & FORS1 \\
20040812 & 53229.39 & 338.2 & $V$ & $3 \times 300$ & 1.03 & 0.74 & FORS1 \\
20040812 & 53229.40 & 338.2 & $R$ & $2 \times 420$ & 1.01 & 0.75 & FORS1 \\
20040812 & 53229.41 & 338.2 & $I$ & $1 \times 500$ & 1.01 & 0.70 & FORS1 \\
20040813 & 53230.36 & 339.2 & $I$ & $4 \times 500$ & 1.08 & 0.78 & FORS1 \\
20040813 & 53230.39 & 339.2 & $R$ & $2 \times 420$ & 1.03 & 0.76 & FORS1 \\
20050207 & 53408.03 & 516.8 & $I$ & $12 \times 240$ & 1.13 & 1.13 & FORS2 \\
20050207 & 53408.08 & 516.9 & $R^{a}$ & $3 \times 600$ & 1.37 & 0.93 & FORS2 \\
20050207 & 53408.09 & 516.9 & $V$ & $3 \times 400$ & 1.49 & 0.98 & FORS2 \\
20050304 & 53433.02 & 541.8 & $U^{a}$ & $3 \times 1140$ & 1.48 & 1.24 & FORS2 \\
20050804 & 53586.40 & 695.2 & $R$ & $2 \times 900$ & 1.04 & 0.70 & FORS1 \\
20050807 & 53589.41 & 698.2 & $I$ & $2 \times 900$ & 1.02 & 0.75 & FORS1 \\
20050808 & 53590.31 & 699.1 & $I$ & $4 \times 900$ & 1.39 & 0.82 & FORS1 \\
20050808 & 53590.34 & 699.1 & $B$ & $5 \times 540$ & 1.19 & 0.82 & FORS1 \\
20050809 & 53591.36 & 700.2 & $R$ & $3 \times 900$ & 1.12 & 0.62 & FORS1 \\
20050810 & 53592.39 & 701.2 & $V$ & $3 \times 480$ & 1.03 & 0.71 & FORS1 \\
\hline
\end{tabular}

${ }^{a}$ The $U$ and $R$ filters on FORS2 are slightly different than the ones on FORS1.

Table A.2. VLT late-time NIR observations of SN 2003hv.

\begin{tabular}{ccccccc}
\hline \hline $\begin{array}{c}\text { Date } \\
\text { (UT) }\end{array}$ & $\begin{array}{c}\text { MJD } \\
\text { (days) }\end{array}$ & $\begin{array}{c}\text { Phase } \\
\text { (days) }\end{array}$ & Filter & $\begin{array}{c}\text { Exposure }^{a} \\
(\mathrm{~s})\end{array}$ & Airmass & $\begin{array}{c}\text { Seeing } \\
(\text { arcsec) }\end{array}$ \\
\hline 20040818 & 53235.37 & 344.2 & $J_{s}$ & $30 \times 4 \times 24$ & 1.04 & 0.55 \\
20040829 & 53246.25 & 355.1 & $K_{s}$ & $10 \times 6 \times 30$ & 1.37 & 0.47 \\
20040829 & 53246.29 & 355.1 & $H$ & $10 \times 6 \times 59$ & 1.17 & 0.75 \\
20040829 & 53246.32 & 355.1 & $K_{s}$ & $10 \times 6 \times 30$ & 1.07 & 0.57 \\
20040829 & 53246.39 & 355.2 & $H$ & $10 \times 6 \times 30$ & 1.00 & 0.75 \\
20040830 & 53247.26 & 356.1 & $J_{s}$ & $30 \times 4 \times 23$ & 1.29 & 0.77 \\
20040830 & 53247.30 & 356.1 & $K_{s}$ & $10 \times 6 \times 30$ & 1.13 & 0.50 \\
20050124 & 53394.02 & 502.8 & $K_{s}$ & $10 \times 6 \times 60$ & 1.03 & 0.47 \\
20050124 & 53394.09 & 502.9 & $H$ & $10 \times 6 \times 30$ & 1.24 & 0.74 \\
20050224 & 53425.99 & 534.8 & $K_{s}$ & $10 \times 6 \times 30$ & 1.18 & 0.62 \\
$20050225^{b}$ & 53426.99 & 535.8 & $J_{s}$ & $30 \times 4 \times 46$ & 1.19 & 0.83 \\
20050227 & 53428.99 & 537.8 & $H$ & $10 \times 6 \times 30$ & 1.22 & 0.55 \\
20050228 & 53429.99 & 538.8 & $J_{s}$ & $30 \times 4 \times 23$ & 1.22 & 0.56 \\
20051013 & 53656.18 & 765.0 & $J_{s}$ & $30 \times 4 \times 46$ & 1.11 & 0.70 \\
20051013 & 53656.35 & 765.2 & $J_{s}$ & $30 \times 4 \times 23$ & 1.14 & 0.56 \\
20051014 & 53657.21 & 766.0 & $J_{s}$ & $30 \times 4 \times 46$ & 1.04 & 0.54 \\
20051014 & 53657.32 & 766.1 & $H$ & $10 \times 6 \times 60$ & 1.05 & 0.72 \\
20051015 & 53658.31 & 767.1 & $H$ & $10 \times 6 \times 30$ & 1.04 & 0.40 \\
\hline
\end{tabular}

${ }^{a}$ Detector integration time (DIT) $\times$ number of DITs per exposure $\times$ number of exposures.

${ }^{b}$ Image not included in the final photometry as it is a poor image and gives bad results.

Table A.3. HST observations of SN $2003 \mathrm{hv}^{a}$.

\begin{tabular}{cccccc}
\hline \hline $\begin{array}{c}\text { Date } \\
\text { (UT) }\end{array}$ & $\begin{array}{c}\text { MJD } \\
\text { (days) }\end{array}$ & $\begin{array}{c}\text { Phase } \\
\text { (days) }\end{array}$ & Instrument & Filter & $\begin{array}{c}\text { Exposure } \\
(\mathrm{s})\end{array}$ \\
\hline 20040710 & 53196.55 & 305.4 & ACS & $F 555 W$ & 480 \\
20040710 & 53196.56 & 305.4 & ACS & $F 814 W$ & 720 \\
20040712 & 53198.82 & 307.6 & ACS & $F 435 W$ & 840 \\
20040712 & 53198.83 & 307.6 & ACS & $F 625 W$ & 360 \\
20040716 & 53202.75 & 311.6 & ACS & $F 435 W$ & 840 \\
20040716 & 53202.76 & 311.6 & ACS & $F 625 W$ & 360 \\
20041115 & 53324.71 & 433.5 & ACS & $F 555 W$ & 480 \\
20041115 & 53324.72 & 433.5 & ACS & $F 814 W$ & 720 \\
20051103 & 53677.31 & 786.1 & NICMOS & $F 160 W$ & $16 \times 640$ \\
\hline \multicolumn{7}{c}{ Observations not conducted due to guide-star failure are not included. }
\end{tabular}

